\title{
On the effects of the degree of discretion in reporting managerial performance
}

\author{
Anja De Waegenaere · Jacco L. Wielhouwer
}

(C) The Author(s) 2010. This article is published with open access at Springerlink.com

\begin{abstract}
We consider a principal-agent setting in which a manager's compensation depends on a noisy performance signal, and the manager is granted the right to choose an (accounting) method to determine the value of the performance signal. We study the effect of the degree of such reporting discretion, measured by the number of acceptable methods, on the optimal contract, the expected cost of compensation, and the manager's expected utility. We find that a minimal degree of discretion may be necessary for successful contracting. We also find that while an increase in reporting discretion never harms the manager, the effect on the expected cost of compensation is more subtle. We identify three main effects of increased reporting discretion and characterize the conditions under which the aggregate of these three effects will lead to a higher or lower cost of compensation. Finally, we find that when reporting discretion induces costly effort on the part of the manager, the optimal degree of discretion can be higher than when it is costless.
\end{abstract}

\footnotetext{
A. De Waegenaere $(\varangle)$

Center for Economic Research,

Department of Accounting and Department of Econometrics

and Operations Research, Tilburg University, P.O. Box 90153,

5000 LE Tilburg, The Netherlands

e-mail: a.m.b.DeWaegenaere@uvt.nl

J. L. Wielhouwer

Department of Accounting, Faculty of Economics,

VU University Amsterdam, De Boelelaan 1105,

1081 HV Amsterdam, The Netherlands

e-mail: jwielhouwer@feweb.vu.nl
} 
Keywords Managerial compensation · Reporting flexibility ·

Principal-agent models

\section{Introduction}

We consider a principal who contracts with a risk- and effort-averse manager in order to motivate him to deliver the desired effort level. Since the effort provided by the manager is not directly observable, the principal contracts on the basis of a noisy signal, e.g. based on accounting numbers. Accounting standards such as Generally Accepted Accounting Principles, however, usually offer a variety of acceptable accounting methods (e.g., LIFO vs. FIFO, accelerated vs straight line depreciation, etc.). It has been demonstrated in several settings that it may be optimal to grant a manager the discretion to choose an accounting method, even when his compensation depends on performance measures derived from reported accounting numbers. Demski et al. (1984) show that when accounting method choice is verifiable, delegating the choice to the manager may be optimal because by motivating the manager to use a different accounting method for different realizations of his private information, the manager's information rent is reduced. Verrecchia (1986) considers a setting where accounting method choice is partially unverifiable, and shows that even when the principal has the option to implicitly eliminate reporting flexibility by affecting the attractiveness of the acceptable reporting alternatives, it is in general not optimal to do so. Ozbilgin and Penno (2008) consider a principal-agent model with a set of ex-ante equivalent performance measurement methods, and find that delegating the choice of measurement method to the manager is optimal if he is sufficiently risk averse.

Given these various conditions under which delegating accounting method choice (or, more generally, performance measurement method) to the manager is optimal, and given the ongoing debate on the "desired" degree of flexibility in GAAP, it is relevant and important to investigate the effect of the degree of reporting flexibility on the internal agency problem. Prior literature shows that risk aversion plays a crucial role in understanding the effect of increased reporting flexibility on the expected cost of compensation. Demski (1998) considers a multi-period model where the manager has private information and can manipulate earnings numbers. He shows that the expected cost of compensation when the manager is motivated to manipulate earnings numbers can be lower than in a situation where he has no private information, so that results can only be reported truthfully. The underlying reason is that the manager can only manipulate the performance signal in case the desirable effort level is delivered, and allowing for manipulation reduces the manager's risk. Ozbilgin and Penno (2008) show that when the manager has the discretion to choose the performance measurement method, increased reporting flexibility (as measured by the number of acceptable performance measurement methods) decreases the expected cost of compensation if the manager is sufficiently risk averse. Their setting has no information asymmetry other than the manager's action and measurement choices. These results show that more reporting flexibility for the manager, either through diversity in acceptable measurement methods (as in Ozbilgin and Penno 2008) or through allowed earnings manipulation 
(as in Demski 1998) can be beneficial to the principal since it reduces the manager's compensation risk. ${ }^{1}$

In this paper we take a principal-agent approach similar to Ozbilgin and Penno (2008), in which the manager's compensation depends on a noisy performance signal, and the manager is granted the right to choose an (accounting) method to determine the value of the performance signal. We study the effect of the degree of reporting flexibility, measured by the number of acceptable measurement methods, on the expected cost of compensation and on the manager's expected utility. ${ }^{2}$ Our results complement and extend theirs in several directions. First, we show that a minimal level of reporting flexibility may be necessary for the existence of an optimal contract, i.e., if incentive problems cannot be resolved at finite cost, an increase in the degree of reporting flexibility can be sufficient to solve this problem. Specifically, when the principal faces a hard agency problem because an unfavorable signal carries little information regarding the manager's action choice, and because the level of utility that the manager derives from compensation cannot be made arbitrarily high by increasing the level of the bonus in case of a favorable signal, it is impossible to design a contract that motivates the manager to deliver high effort. Increasing the degree of reporting flexibility mitigates this problem because it increases the informativeness of an unfavorable signal. Thus, if a manager cannot be motivated to deliver high effort by increasing the level of the bonus, increasing the level of reporting discretion may be an alternative means to resolve incentive conflicts.

Second, whether the limited liability constraint is binding in our setting is endogenous and depends on the degree of reporting flexibility. We distinguish two critical values of the degree of reporting flexibility. The first critical value determines whether the manager will earn a limited liability rent. The second critical value determines whether increased reporting flexibility makes it easier or more difficult to prevent shirking, i.e., whether a higher bonus is required to motivate high effort. As long as the degree of flexibility does not exceed either of these two critical values, higher reporting flexibility yields a lower expected cost of compensation, even though it does not affect the manager's expected utility. Above the two threshold values, increased reporting flexibility is strictly beneficial to the manager, but harmful to the principal. For intermediate degrees of reporting flexibility, the effect is ambiguous. We show that increased reporting flexibility may then be socially optimal in the sense that it makes both the principal and the manager strictly better off.

Third, determining performance signals from multiple measurement methods may require costly effort on the part of the manager. We investigate the effect of such flexibility induced costly reporting effort on the expected compensation cost. We find that, compared to the case where reporting effort is costless, offering reporting flexibility may be more attractive, i.e., the optimal degree of reporting flexibility can be higher, when flexibility induces costs. Moreover, for sufficiently large degrees of reporting

\footnotetext{
1 Penno (2005) considers a manager who can choose between $N$ performance measurement signals that are i.i.d. exponentially distributed, and shows that the expected cost of compensation is independent of $N$. This remarkable result is due to the nature of the exponential distribution.

2 Ozbilgin and Penno (2008) distinguishes settings in which the discretion to choose the method rests with the principal and settings where it rests with the manager. The focus in our paper is on the latter.
} 
flexibility, the expected cost of compensation can be strictly lower when reporting induces costly effort, than when it is costless. This occurs for two reasons. When reporting costs are not too high, the manager will, in expectation, engage in more costly reporting effort when low effort was delivered than when high effort was delivered. Thus, costly effort associated with determining performance signals makes low effort relatively less attractive, so that it becomes easier to motivate the manager to deliver high effort. Second, when the degree of reporting flexibility is sufficiently high, compensation for costly reporting effort may lead to a reduction in the limited liability rent. The combination of these two effects implies that higher degrees of flexibility can be more attractive when flexibility induces costly reporting effort, than when it is costless.

Although related, the problem studied in this paper differs in several ways from the literature on equilibrium earnings management when the Revelation Principle fails to hold due to, e.g., restricted communication, lack of commitment, or contracting restrictions. There, the focus is on settings where the manager has private information and may be able to manage earnings in a way that would not be accepted if detected by an audit system. The issue is then whether motivating rejection of earnings management is optimal. It has been demonstrated that allowing for, and motivating, manipulation of performance measures may be beneficial to the principal in situations where manipulation requires costly effort Demski et al. (2004), Liang (2004) or when there is limited commitment Arya et al. (1998). In our setting, there is no private information (other than the action and measurement method choice) and all available measurement methods are equally acceptable. The issue is therefore not whether the manager should be motivated to choose a particular method. Rather, the focus is on the effect of diversity in measurement methods on the expected cost of compensation, given that the manager can strategically choose any method from the set of acceptable methods.

The rest of the paper is organized as follows. In Sect. 2 we present the model. In Sect. 3 we derive the optimal contract conditional on the number of acceptable accounting methods. In Sect. 4 we study the effect of increased reporting flexibility on the optimal contract, the expected cost of compensation, and the manager's expected utility. Section 5 discusses the implications of reporting flexibility for both the principal and the manager. Section 6 investigates the effect of costs associated with reporting flexibility. Section 7 concludes. All proofs are deferred to the Appendix.

\section{The model}

We consider a principal who contracts with a risk- and effort-averse manager in order to motivate him to deliver the desired effort level. Since the effort provided by the manager is not directly observable, the principal contracts on the basis of a noisy signal. The model is similar to the models in Ozbilgin and Penno (2008), Penno (2005). Specifically, there is managerial reporting flexibility in the sense that there are a number of different noisy performance signals, each resulting from equally acceptable measurement methods. Our focus is on the case where the choice of accounting method is delegated to the manager, i.e., the manager has the discretion to choose a measurement 
method, and report only the corresponding signal to the principal. ${ }^{3}$ The choice occurs ex post, i.e., after all the signals have been realized.

There are two effort levels $a \in\left\{a_{H}, a_{L}\right\}$, and a set of $N$ equally acceptable measurement methods. Each method yields a signal that can take two values $y \in\left\{y_{H}, y_{L}\right\}$, with $y_{H}>y_{L}$. The signals resulting from the $N$ different measurement methods are independent and identically distributed random variables $y^{i}, i=1, \ldots, N$, for which the probability distribution is determined by the action chosen by the manager in the following way:

$$
\begin{aligned}
& P\left\{y^{i}=y_{H} \mid a=a_{H}\right\}=1-p, \\
& P\left\{y^{i}=y_{L} \mid a=a_{H}\right\}=p, \\
& P\left\{y^{i}=y_{H} \mid a=a_{L}\right\}=1-q, \\
& P\left\{y^{i}=y_{L} \mid a=a_{L}\right\}=q .
\end{aligned}
$$

Without loss of generality we assume that $0<p<q<1$, i.e. the probability of outcome $y_{L}$ is higher under $a_{L}$ than under $a_{H}$. This implies that the monotone likelihood ratio property (MLRP) holds, i.e., if $a_{H}$ is the desirable action, $y_{H}$ is a "good" signal, and $y_{L}$ is a "bad" signal.

The principal is risk neutral; the manager is a risk- and effort-averse expected utility maximizer. His utility depends on the level of compensation $x$, and the effort level $a$, and is given by:

$$
u(x, a)=-e^{-\rho(x-c(a))}, \text { for } x \in \mathbb{R}, \text { and } a \in\left\{a_{L}, a_{H}\right\},
$$

where $\rho>0$ represents the degree of risk aversion, and $c(a)$ represents the monetary equivalent of the disutility associated with effort level $a$ (see, e.g., Grossman and Hart 1983). Because the manager is effort-averse, we let $c_{H}=c\left(a_{H}\right)>c_{L}=c\left(a_{L}\right)$.

The timeline is as follows:

- Date 0: The principal specifies the level of discretion $N$, and the levels of compensation that will be paid to the manager in case $y_{H}$, respectively $y_{L}$, is reported. The manager decides to accept or reject the contract. If the manager accepts the contract, he then chooses his effort level $a \in\left\{a_{H}, a_{L}\right\}$.

- Date 1: The manager determines the value $y^{i} \in\left\{y_{H}, y_{L}\right\}$ of the signal resulting from the $i$ th acceptable performance measurement method, for $i=1, \ldots, N$, and reports one signal $\widehat{y} \in\left\{y^{i} ; i=1, \ldots, N\right\}$ to the principal. Compensation is paid and the game ends.

\footnotetext{
3 Alternatively, the principal could require the manager to report all $N$ signals instead of only one. As argued in Ozbilgin and Penno (2008), Penno (2005), however, reported performance signals are typically not uncontestable until they are subjected to a costly verification process. Therefore, while requiring the manager to report all signals instead of just one would give the principal the option to use more than one performance signal, it would also increase the cost to the principal associated with verifying reported signals.
} 
Without loss of generality, we focus on the compensation scheme needed to motivate the manager to take action $a_{H} \cdot{ }^{4}$ Then, similarly to (Arya et al. 1992; Dye and Magee 1991; Ozbilgin and Penno 2008), the principal needs to minimize the expected cost of inducing the agent to choose action $a_{H}$, taking into account his self-interested behavior with respect to his action and reporting choices. Specifically, for any given level of reporting flexibility $N$, the principal determines the corresponding compensation levels such that the expected cost of compensation is minimized, under the constraints that: i) the manager reports the most favorable signal (i.e., the one that maximizes his compensation) among the set of $N$ acceptable signals $y^{i}, i=1, \ldots, N$, ii) providing high effort yields a higher expected utility than providing low effort (incentive compatibility), iii) staying with the firm and accepting the contract is preferable to the first best alternative (individual rationality), and, iv) compensation is nonnegative (limited liability). ${ }^{5}$

Let us denote $s(y, N)$ for the compensation received in case $N$ measurement methods are available, and signal $y$ is reported. Moreover, let $\bar{M}$ denote the manager's reservation utility. Then, for any given level of reporting flexibility $N$, the following optimization problem needs to be solved:

$$
\begin{array}{ll}
\min & \mathbb{E}\left[s(\widehat{y}, N) \mid a=a_{H}\right] \\
& \widehat{y} \in \arg \max _{y \in\left\{y^{1}, \ldots, y^{N}\right\}} s(y, N) \\
\text { s.t. } & \mathbb{E}\left[u(s(\widehat{y}, N), a) \mid a=a_{H}\right] \geqslant \bar{M} \\
& \mathbb{E}\left[u(s(\widehat{y}, N), a) \mid a=a_{H}\right] \geqslant \mathbb{E}\left[u(s(\widehat{y}, N), a) \mid a=a_{L}\right] \\
& s\left(y_{H}, N\right) \geqslant 0, s\left(y_{L}, N\right) \geqslant 0
\end{array}
$$

It now remains to specify how the probability distribution of the reported signal $\hat{y}$ depends on the degree of reporting flexibility $N$, and the action choice $a$. Clearly,

$$
\begin{aligned}
& s\left(y_{H}, N\right) \geqslant s\left(y_{L}, N\right) \Longrightarrow \widehat{y}=\max \left\{y^{i} ; i=1, \ldots, N\right\} \\
& s\left(y_{H}, N\right) \leqslant s\left(y_{L}, N\right) \Longrightarrow \widehat{y}=\min \left\{y^{i} ; i=1, \ldots, N\right\} .
\end{aligned}
$$

It is easy to verify that due to the MLRP, the optimal contract when $\widehat{y}=\min \left\{y^{i} ; i=\right.$ $1, \ldots, N\}$ satisfies $s\left(y_{H}, N\right)>s\left(y_{L}, N\right)$. Therefore, $\widehat{y}=\max \left\{y^{i} ; i=1, \ldots, N\right\}$, i.e., the reported signal $\widehat{y}$ equals $y_{H}$ if for at least one measurement method it holds that $y^{i}=y_{H}$, and equals $y_{L}$ otherwise. Consequently, as in Ozbilgin and Penno (2008), Penno (2005), the probability distribution of the reported signal under high effort depends on the number of acceptable measurement alternatives in the

\footnotetext{
4 It is easily verified that, for all $N$, the cost minimizing compensation scheme that motivates the manager to take action $a_{L}$ when $N$ methods are available consists of a flat wage of $M+c_{L}$.

5 As argued in Lewis (1980), the penalties that the principal may impose on the manager are typically limited by bankruptcy and/or legal liability laws. While limited liability is sometimes interpreted as an (imperfect) substitute for risk aversion, ignoring either risk aversion or limited liability may significantly affect the second best solution (see, e.g., Holmström 1979; Lewis 1980). Other papers that do include both risk aversion and limited liability include, e.g., also Mookherjee and Png (1989), Ozbilgin and Penno (2008), Penno (2005), Rasmusen (1987).
} 
following way:

$$
\begin{aligned}
P & \left\{\widehat{y}=y_{L} \mid a=a_{H}\right\} \\
& =P\left(\max \left\{y^{i} ; i \in\{1, \ldots, N\}\right\}=y_{L} \mid a=a_{H}\right) \\
& =P\left(y^{1}=y_{L}, y^{2}=y_{L}, \ldots, y^{N}=y_{L} \mid a=a_{H}\right) \\
& =P\left(y^{1}=y_{L} \mid a=a_{H}\right) P\left(y^{2}=y_{L} \mid a=a_{H}\right) \cdots P\left(y^{N}=y_{L} \mid a=a_{H}\right) \\
& =p^{N},
\end{aligned}
$$

and,

$$
P\left\{\widehat{y}=y_{H} \mid a=a_{H}\right\}=1-p^{N} .
$$

Similarly, for low effort:

$$
\begin{aligned}
& P\left\{\widehat{y}=y_{H} \mid a=a_{L}\right\}=1-q^{N}, \\
& P\left\{\widehat{y}=y_{L} \mid a=a_{L}\right\}=q^{N} .
\end{aligned}
$$

For notational convenience, we denote $s\left(y_{H}, N\right)=s_{H}(N)$ and $s\left(y_{L}, N\right)=s_{L}(N)$, for the compensation levels when $N$ measurement methods are available, and in case signal $y_{H}$ or $y_{L}$ is reported, respectively. Moreover, we let $M=-\frac{1}{\rho} \ln (-\bar{M})$ denote the monetary equivalent of the reservation utility $\bar{M}$. Then, (3) and (7)-(10) imply that optimization problem (4) is equivalent to:

$$
\begin{array}{ll}
\min & p^{N} \cdot s_{L}(N)+\left(1-p^{N}\right) \cdot s_{H}(N) \\
\text { s.t. } & p^{N} \cdot e^{-\rho\left(s_{L}(N)-c_{H}\right)}+\left(1-p^{N}\right) \cdot e^{-\rho\left(s_{H}(N)-c_{H}\right)} \\
& \leqslant q^{N} \cdot e^{-\rho\left(s_{L}(N)-c_{L}\right)}+\left(1-q^{N}\right) \cdot e^{-\rho\left(s_{H}(N)-c_{L}\right)} \\
& p^{N} \cdot e^{-\rho\left(s_{L}(N)-c_{H}\right)}+\left(1-p^{N}\right) \cdot e^{-\rho\left(s_{H}(N)-c_{H}\right)} \leqslant e^{-\rho M} \\
& s_{L}(N) \geqslant 0, s_{H}(N) \geqslant 0 .
\end{array}
$$

Our goal is to study the effect of the degree of reporting flexibility $N$ on agency costs. In the next section we first determine how the principal optimally conditions the contractually committed payments on the degree of reporting flexibility $N$. This will then allow us to characterize conditions under which allowing higher degrees of flexibility is beneficial to the principal.

\section{Contract payments conditional on $N$}

The following theorem shows that a minimal degree of reporting discretion may be necessary in order to be able to write a contract that motivates the agent to perform high effort. It also shows how the principal optimally conditions the contractually committed payments in case of a favorable and an unfavorable signal, respectively, on the degree of reporting flexibility $N$. 
Theorem 1 A minimal level of reporting flexibility is necessary for the existence of an optimal contract. Specifically, an optimal compensation contract exists iff

$$
N>\frac{\rho\left(c_{H}-c_{L}\right)}{\ln q-\ln p} .
$$

Then, the limited liability constraint is binding iff $N>N^{*}$, where

$$
N^{*}=\max \left\{N \in \mathbb{N}: \frac{1-q^{N}}{1-p^{N}} \leqslant \frac{1-e^{-\rho\left(M+c_{L}\right)}}{1-e^{-\rho\left(M+c_{H}\right)}}\right\} .
$$

Moreover:

(i) If $N \leqslant N^{*}$, the optimal compensation scheme is given by

$$
\begin{aligned}
s_{L}(N) & =-\frac{1}{\rho} \ln \frac{\left(1-p^{N}\right) e^{-\rho\left(M+c_{L}\right)}-\left(1-q^{N}\right) e^{-\rho\left(M+c_{H}\right)}}{q^{N}-p^{N}}, \\
s_{H}(N) & =-\frac{1}{\rho} \ln \frac{q^{N} e^{-\rho\left(M+c_{H}\right)}-p^{N} e^{-\rho\left(M+c_{L}\right)}}{q^{N}-p^{N}} .
\end{aligned}
$$

(ii) If $N>N^{*}$, the optimal compensation scheme is given by

$$
\begin{aligned}
& s_{L}(N)=0, \\
& s_{H}(N)=-\frac{1}{\rho} \ln \frac{q^{N} e^{-\rho\left(M+c_{H}\right)}-p^{N} e^{-\rho\left(M+c_{L}\right)}}{\left(1-p^{N}\right) e^{-\rho\left(M+c_{L}\right)}-\left(1-q^{N}\right) e^{-\rho\left(M+c_{H}\right)}} .
\end{aligned}
$$

The above theorem shows that a minimal degree of reporting flexibility may be necessary to be able to resolve incentive conflicts at finite cost. The intuition is as follows. Providing high effort yields a higher probability of getting the bonus, but also yields a higher disutility from effort. Delivering high effort can be made more attractive by increasing the level of the bonus. However, the level of the utility associated with compensation received in case of a favorable signal cannot be made arbitrarily high by increasing the size of the bonus (i.e., the utility function has a bliss level). This implies that when the agency problem is "severe" because an unfavorable signal carries little information regarding the manager's action choice, the agent cannot be motivated to provide high effort. Specifically, if the manager is constrained to using a specific measurement method (i.e., when $N=1$ ), then there does not exist an optimal contract if the likelihood ratio of an unfavorable signal is low relative to the manager's risk-adjusted cost differential, i.e., if $\frac{q}{p}<e^{\rho\left(c_{H}-c_{L}\right)}$. Allowing more flexibility by increasing the number of available methods solves this problem, because it makes a low signal more informative. Indeed, a contract that resolves incentive problems exists if the manager can choose amongst $N>\frac{\rho\left(c_{H}-c_{L}\right)}{\ln q-\ln p}$ performance measurement methods, because then the likelihood ratio of an unfavorable signal increases to $\frac{q^{N}}{p^{N}}=\left(\frac{q}{p}\right)^{N}>e^{\rho\left(c_{H}-c_{L}\right)}$. Thus, if a manager cannot be motivated to deliver high effort by increasing the level of the bonus, increasing the level of reporting discretion may be an alternative means 

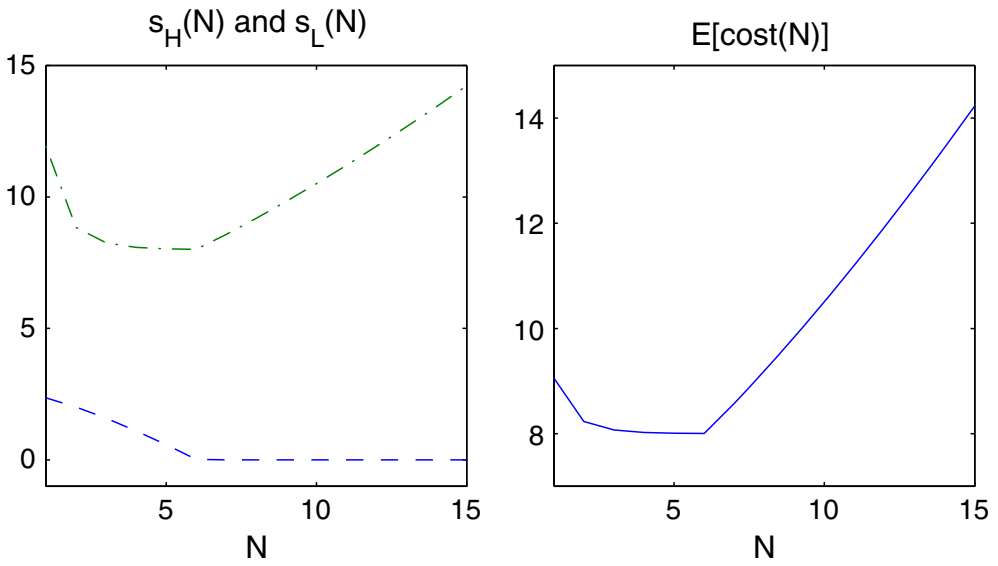

Fig. 1 Optimal values of $s_{H}(N)$ (dashed-dotted) and $s_{L}(N)$ (dashed) (left panel) and expected cost (right panel), as a function of $N$ for $\rho=0.1, c_{L}=0, c_{H}=5, M=3, p=0.3, q=0.9$

to resolve incentive conflicts. If, in contrast, the utility of compensation in case of a favorable signal can be made arbitrarily high by increasing the level of the bonus, as is the case for example for the power utility function considered in Ozbilgin and Penno (2008), then there always exists a level of the bonus that makes it optimal for the manager to perform high effort. ${ }^{6}$

Theorem 1 also shows that the way in which the optimal contract is affected by the level of reporting flexibility depends crucially on whether the threshold value $N^{*}$ is exceeded, or, equivalently, whether the limited liability constraint is binding. It is therefore intuitively clear that this threshold value can also play a dominant role in the effect of reporting flexibility on the expected cost of compensation.

This is illustrated in Fig. 1. It shows the payoffs $s_{L}(N)$ and $s_{H}(N)$ and the expected cost of the optimal contract, as a function of the number of alternative measurement methods. We see that the optimal payoffs as well as the expected cost of compensation first decrease and then increase as $N$ increases. Although in the setting in Fig. 1 the limited liability constraint becomes binding at $N^{*}=6$, and the expected cost of compensation decreases (increases) in $N$ for $N \leqslant 6(N>6)$, we will show in the sequel that this is not the general pattern.

\section{The effect of increased reporting flexibility}

In this section we determine the effect of an increase in the level of reporting flexibility on the level of compensation and the size of the bonus (Subsect. 4.1), on the manager's expected utility (Subsect. 4.2), and on the expected cost of compensation (Subsect. 4.3).

\footnotetext{
6 We thank an anonymous reviewer for this insight.
} 


\subsection{The effect on the optimal contract}

For the sake of intuition, we view the compensation package as consisting of a level of compensation $s_{L}(N)$, to which a bonus $s_{H}(N)-s_{L}(N)$ is added in case of a high report. We also introduce a second threshold value $\widetilde{N}$, which is defined as follows:

$$
\widetilde{N}=\frac{\ln \left(\frac{\ln p}{\ln q}\right)+\rho\left(c_{H}-c_{L}\right)}{\ln q-\ln p} .
$$

In the following theorem we determine the effect of the level of reporting flexibility $(N)$ on the two levels of compensation $s_{L}(N)$ and $s_{H}(N)$, as well as on the size of the bonus (the difference between the two levels of compensation), where the critical values $N^{*}$ and $\widetilde{N}$ are as defined in (12) and (17), respectively.

Theorem 2 For the optimal compensation contract, the following holds:

(i) $s_{H}(N)$ is decreasing in $N$ for $N \leqslant \max \left\{N^{*}, \widetilde{N}\right\}$, and increasing in $N$ for $N>$ $\max \left\{N^{*}, \widetilde{N}\right\}$,

(ii) $s_{L}(N)$ is decreasing in $N$ for $N \leqslant N^{*}$, and $s_{L}(N)=0$ for $N>N^{*}$.

(iii) $s_{H}(N)-s_{L}(N)$ is decreasing in $N$ for $N \leqslant \widetilde{N}$, and increasing in $N$ for $N>\widetilde{N}$.

The above theorem shows that the optimal contract depends crucially on the two critical values, $\widetilde{N}$ and $N^{*}$. First, regardless of whether the limited liability constraint is binding or not, the critical level $\widetilde{N}$ determines whether an increase in $N$ makes incentive problems more severe, or equivalently, whether a higher bonus is required to motivate high effort. As long as $N \leqslant \widetilde{N}$, an increase in the degree of reporting flexibility makes incentive problems less severe, so that the size of the bonus can be decreased. The opposite holds when $N$ is higher than the critical level $\widetilde{N}$. The intuition is as follows. The effect of an increase in $N$ on the level of the bonus depends on whether the increase makes it easier or more difficult to motivate the agent to provide high effort (i.e., to satisfy the incentive compatibility constraint). An increase in $N$ increases the probability that the bonus is achieved under high effort (i.e., it increases $1-p^{N}$ ). However, it also increases the probability that the bonus is achieved when low effort is provided (i.e., it increases $1-q^{N}$ ), so that shirking becomes more attractive. Whether the bonus needs to be increased or can be decreased in response to a higher degree of reporting discretion depends on which of these two effects is dominant. When $N$ is sufficiently low, an increase in reporting flexibility has a stronger effect on $1-p^{N}$ than on $1-q^{N}$, and vice versa when $N$ is sufficiently high. Therefore, there exists a threshold value such that a lower (higher) bonus is needed if the level of discretion is below (above) the threshold value. The threshold value depends on the severity of the agency problem. If the problem is more "severe" because of either a high cost differential, or a high degree of risk aversion, then an increase in the probability of getting the bonus affects the manager's expected utility under high 
effort more than under low effort. Therefore, the threshold value $\widetilde{N}$ is increasing in $\rho\left(c_{H}-c_{L}\right){ }^{7}$

Second, the critical level $N^{*}$ determines whether the limited liability constraint is binding. An increase in $N$ increases $1-p^{N}$, i.e., there is a higher probability of getting the bonus. It therefore allows for a lower level of compensation in case of an unfavorable signal $\left(s_{L}(N)\right)$. This eventually makes the limited liability constraint binding. As long as the limited liability constraint is not binding (i.e., $N \leqslant N^{*}$ ), both levels of compensation can be decreased when reporting flexibility increases. When the lowerbound on compensation becomes binding $\left(N>N^{*}\right)$, the compensation in case of an unfavorable signal needs to be fixed at its minimal level. Consequently, the size of the bonus can only be affected by changing the level of the compensation in case of a favorable report. It needs to be increased when $N>\widetilde{N}$, but can be decreased when $N \leqslant \widetilde{N}$.

\subsection{The effect on the manager's expected utility}

In this section we study the effect of an increase in the degree of reporting flexibility on the manager's expected utility. Let us therefore denote $C E(N)$ for the manager's certain equivalent as a function of the number of alternative measurement methods, $N$, i.e., $C E(N)$ is such that

$$
\begin{aligned}
u\left(C E(N), a_{H}\right) & =\mathbb{E}\left[u(s(\widehat{y}, N), a) \mid a=a_{H}\right] \\
& =\left(1-p^{N}\right) \cdot u\left(s_{H}(N), a_{H}\right)+p^{N} \cdot u\left(s_{L}(N), a_{H}\right),
\end{aligned}
$$

where $s_{H}(N)$ and $s_{L}(N)$ are as defined in Theorem 1 . The following theorem determines the effect of $N$ on the manager's certain equivalent.

Theorem 3 For the manager's certain equivalent, the following holds:

$$
\begin{aligned}
C E(N) & =M+c_{H}, & \text { for } N \leqslant N^{*}, \\
& =\frac{1}{\rho} \ln \frac{\left(1-p^{N}\right) e^{\rho\left(c_{H}-c_{L}\right)}-\left(1-q^{N}\right)}{q^{N}-p^{N}}, & \text { for } N>N^{*} .
\end{aligned}
$$

The certain equivalent is strictly increasing in $N$ for $N>N^{*}$.

The above theorem implies that the manager's utility is not affected by a change in the degree of reporting flexibility $N$ as long as the $N$ does not exceed critical level $N^{*}$. The expected utility of the manager is then equal to his reservation utility. Above the critical level $N^{*}$, the manager starts earning a rent due to the fact that the limited liability constraint becomes binding. Since the rent is increasing in $N$, the manager strictly benefits from increased reporting flexibility.

This is illustrated in Fig. 2. It can be verified that the limited liability constraint becomes binding at $N^{*}=4$, for $c_{L}=0$ and $c_{L}=1$, and at $N^{*}=6$ for $c_{L}=3$. Once

\footnotetext{
7 Because the IC constraint is binding in the optimum (see proof of Theorem 1), the level of the bonus, $s_{H}(N)-s_{L}(N)$, follows from: $p^{N}+\left(1-p^{N}\right) e^{-\rho\left(s_{H}(N)-s_{L}(N)\right)}=\left[q^{N}+\left(1-q^{N}\right) e^{-\rho\left(s_{H}(N)-s_{L}(N)\right)}\right]$ $e^{-\rho\left(c_{H}-c_{L}\right)}$. It can be verified that $\frac{d}{d N}\left(1-p^{N}\right)>\frac{d}{d N}\left(1-q^{N}\right)$ iff $N \leq \ln \left(\frac{\ln p}{\ln q}\right) /(\ln q-\ln p)$ (see proof of Theorem 2 for $\alpha=1$ ).
} 


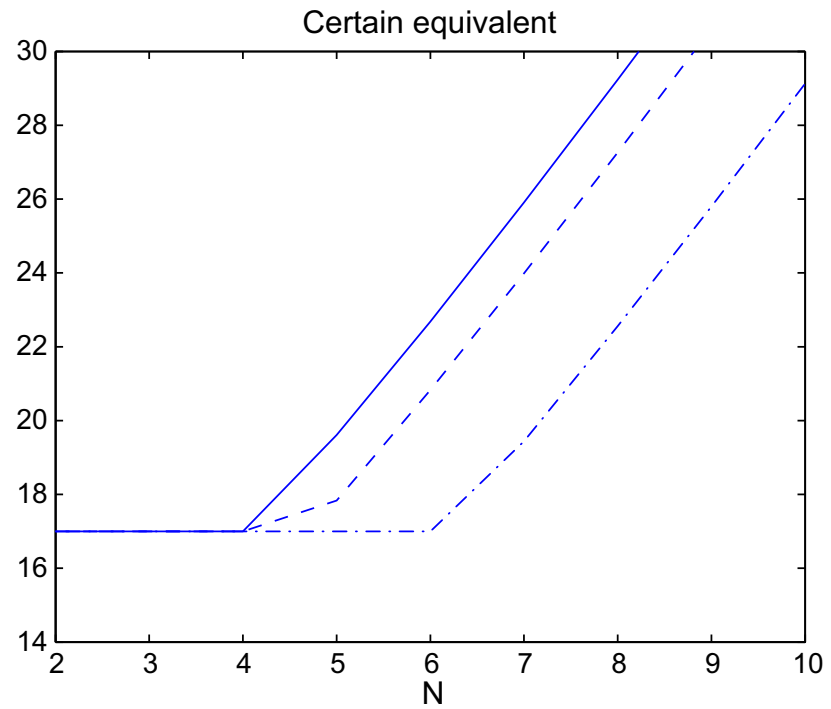

Fig. 2 The manager's certain equivalent, as a function of $N$, for $p=0.3, q=0.7, \rho=0.1, M=10$, $c_{H}=7$, and $c_{L}=0$ (solid line $), c_{L}=1$ (dashed line $)$ and $c_{L}=3$ (dashed-dotted line $)$

the limited liability constraint is binding, the agent earns a limited liability rent. The size of the rent increases when the degree of reporting flexibility increases. The rate of the increase is increasing in the difference between the cost of high and low effort.

\subsection{The effect on the expected cost of compensation}

In this section we study the effect of an increase in the degree of reporting flexibility on the expected cost of compensation, which is given by:

$$
\mathbb{E}\left[s(\widehat{y}, N) \mid a=a_{H}\right]=\left(1-p^{N}\right) \cdot s_{H}(N)+p^{N} \cdot s_{L}(N),
$$

where $s_{H}(N)$ and $s_{L}(N)$ denote the optimal levels of compensation in case $N$ methods are available, as given in Theorem 1. In particular, we characterize conditions under which increased reporting flexibility leads to lower (higher) agency costs.

The analysis in the previous subsections hints at the fact that the effect of reporting flexibility on the expected cost of compensation will be driven by the following three effects:

- The decreased compensation risk effect: because $p^{N}$ is strictly decreasing in $N$, an increase in the degree of reporting flexibility implies that the manager faces less compensation risk because the probability that he will receive the bonus increases. Consequently, the compensation in case of an unfavorable signal can be decreased (Theorem 2 ii).

- The incentive compatibility effect: the bonus can be decreased when $N<\widetilde{N}$ (needs to be increased when $N \geqslant \widetilde{N}$ ) because an increase in reporting flexibility then mitigates (aggravates) incentive problems (Theorem 2 iii). 
- The limited liability effect: when $N \geqslant N^{*}$, the limited liability constraint is binding, and the manager earns a rent which increases in the level of reporting flexibility (Theorem 3).

In isolation, each of these effects is either cost increasing or cost decreasing, but the aggregate effect is non-trivial. For example, when $N<N^{*}$, the limited liability constraint is not binding, and the agency cost equals the risk premium. Then, even when increased flexibility increases the bonus (i.e., when $N>\tilde{N}$ ), the expected cost of compensation does not necessarily increase because the increase in the probability that the bonus is received reduces the manager's compensation risk.

In the sequel we characterize the conditions under which increased reporting flexibility will increase (decrease) the expected cost of compensation. The following theorem shows that for sufficiently high values of $N$, the cost increasing effects of the limited liability rent and increased incentive problems are dominant.

Theorem 4 If $N>\max \left\{N^{*}, \widetilde{N}\right\}$, then the expected cost of compensation is increasing in $N$.

We now focus on the case where $N \leqslant \max \left\{N^{*}, \tilde{N}\right\}$. The following theorem shows that whether a higher degree of reporting flexibility would increase or decrease the expected cost of compensation depends on: the probabilities $p^{N}$ and $q^{N}$, whether $N$ exceeds the threshold value $N^{*}, 8$ and the manager's personal cost factor $\alpha$, which is defined as:

$$
\alpha=e^{-\rho\left(c_{H}-c_{L}\right)} .
$$

The parameter $\alpha$ reflects the severity of the agency problem. A lower value of $\alpha$, e.g., due to a higher degree of risk aversion and/or a bigger difference between the cost of high effort and low effort, ceteris paribus, implies that compensation will be more costly.

Theorem 5 Let $p, q$, and $N \leqslant \max \left\{N^{*}, \tilde{N}\right\}$ be given, and denote

$$
S=\{(u, v) \in[0,1] \times[0,1]:(u-2 u v+v) \ln u-2 v(1-u) \ln v>0\} .
$$

Then, there exists an $\alpha^{*}$ and an $\alpha_{b}^{*}$ such that: ${ }^{9}$

(i) If $N<N^{*}$, an increase in $N$ decreases the expected cost of compensation if $\alpha<\alpha^{*}$ or $\left(p^{N}, q^{N}\right) \notin S$, and increases the expected cost of compensation otherwise.

(ii) If $N^{*} \leqslant N \leqslant \widetilde{N}$, an increase in $N$ decreases (increases) the expected cost of compensation iff $\alpha<\alpha_{b}^{*}\left(\alpha>\alpha_{b}^{*}\right)$.

Figure 3 illustrates the set $S$.

\footnotetext{
${ }^{8}$ Given $p^{N}$ and $q^{N}$, the number of alternative signals $N$ affects the magnitude of the derivative of the expected cost of compensation with respect to $N$, but not its sign.

9 The critical values $\alpha^{*}$ and $\alpha_{b}^{*}$ depend on $p^{N}$ and $q^{N}$. In order to avoid overloaded notation, we do not explicitly denote this dependence, unless it is required for clarity.
} 


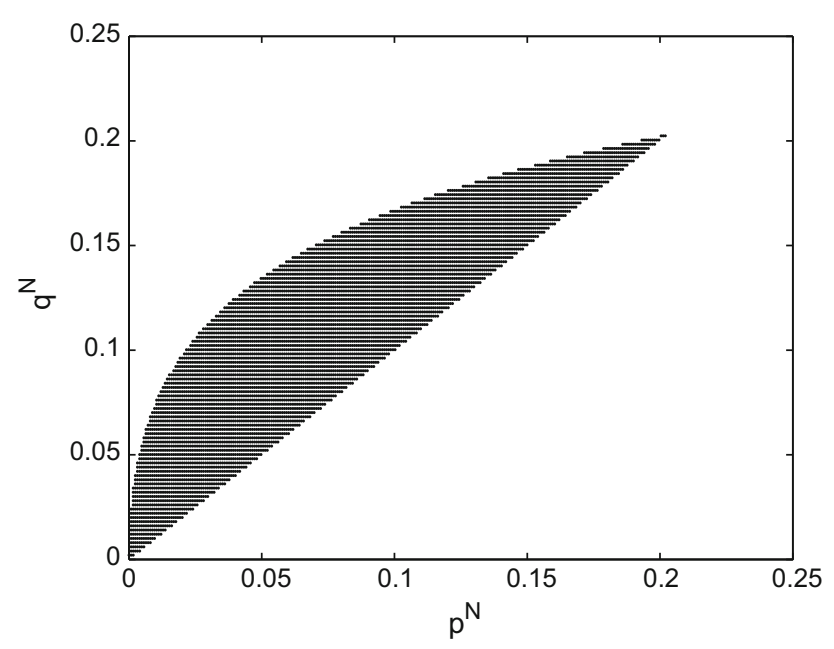

Fig. 3 The combinations of $p^{N}$ and $q^{N}$ for which $\left(p^{N}, q^{N}\right) \in S$

Finally, we discuss the effect of the degree of reporting flexibility on the risk premium, which is given by:

$$
\mathbb{E}\left[s(\widehat{y}, N) \mid a=a_{H}\right]-C E(N)=\left(1-p^{N}\right) \cdot s_{H}(N)+p^{N} \cdot s_{L}(N)-C E(N),
$$

where $s_{H}(N)$ and $s_{L}(N)$ denote the optimal levels of compensation in case $N$ methods are available, as given in Theorem 1 , and $C E(N)$ denotes the manager's certain equivalent, as given in Theorem 3 . The risk premium is equal to the agency cost when $N \leqslant N^{*}$, because then the limited liability constraint is not binding, and the agent's certain equivalent is equal to $M+c_{H}$, the compensation cost in absence of an agency problem. However, when $N>N^{*}$, the limited liability constraint is binding and the agency cost is strictly higher than the risk premium because the agent earns a limited liability rent. Moreover, whereas the agency cost is affected by the limited liability constraint when $N>N^{*}$ (i.e., the agency cost would be lower in absence of the limited liability constraint), this is not the case for the risk premium. Both with and without the liability constraint, the risk premium is equal to the agency cost in absence of the limited liability constraint. This yields the following result.

Theorem 6 Let $S$ be as defined in Theorem 5. Then, there exists an $\alpha^{*}$ such that an increase in $N$ decreases the risk premium if $\alpha<\alpha^{*}$ or $\left(p^{N}, q^{N}\right) \notin S$, and increases the risk premium otherwise.

\section{Implications}

In this section we use Theorems 3 and 5 to study the implications of increased reporting flexibility for both the principal and the manager. We will distinguish four ranges of values of $N$, depending on whether $\widetilde{N}<N^{*}$, or vice versa. Note that $N^{*}$ is decreasing 
in $c_{H}$ and increasing in $c_{L}$, and the opposite holds for $\widetilde{N}$. Moreover, $N^{*}$ is increasing in $M$, and $\widetilde{N}$ is independent of $M$. Therefore, the case $\widetilde{N}<N^{*}$ is more likely to occur when the cost differential $c_{H}-c_{L}$ is high, and/or when the reservation utility $M$ is high.

The case where $N \leqslant \min \left\{N^{*}, \tilde{N}\right\}$.

In this case, the limited liability constraint is not binding (because $N \leqslant N^{*}$ ), and an increase in reporting flexibility mitigates incentive problems and therefore allows for a lower bonus (because $N \leqslant \widetilde{N}$ ). Now it can be verified numerically that: ${ }^{10}$

$$
N \leqslant \widetilde{N} \Longrightarrow \alpha<\alpha^{*}
$$

It therefore follows from Theorem 5 (i) that the expected cost of compensation will be decreasing in $N$. Moreover, since the limited liability constraint is not binding, it follows from Theorem 3 that the manager does not earn a rent. This yields:

Implication 1: If $N$ is sufficiently low $\left(N \leqslant \min \left\{N^{*}, \tilde{N}\right\}\right)$, an increase in the degree of reporting flexibility makes the principal strictly better off, while leaving the manager's utility unaffected.

The case where $\tilde{N} \leqslant N \leqslant N^{*}$.

In this case, the limited liability constraint is still not binding (because $N \leqslant N^{*}$ ), but an increase in reporting flexibility now aggravates incentive problems and thus a higher bonus is required (since $\widetilde{N} \leqslant N$ ). The effect on the expected cost of compensation therefore depends on whether the cost reducing effect of reduced compensation risk outweighs the cost increasing effect of increased incentive problems. It follows from Theorem 5 i) that the aggregate effect of increased reporting flexibility on the expected cost of compensation depends on both the risk aversion/cost parameter $\alpha$, as well as on $\left(p^{N}, q^{N}\right)$. Combined with the result from Theorem 3 , this yields:

Implication 2: If $\widetilde{N} \leqslant N \leqslant N^{*}$, an increase in the degree of reporting flexibility leaves the manager's utility unaffected, and makes the principal better off if $\alpha<a^{*}$ or $\left(p^{N}, q^{N}\right) \notin S$, and worse off otherwise.

Since $S \subset[0,0.2] \times[0,0.2]$, there is a wide range of parameter values for which $\left(p^{N}, q^{N}\right) \notin S$. We illustrate this in Fig. 4 .

For the parameter values in Fig. 4 , it holds that $\widetilde{N}=2$ and $N^{*}=26$. The critical value of $\alpha$ needed to make the increased incentive problems effect dominant, $\alpha^{*}$, decreases for $N \leqslant 5$, and increases for $N>5$. It can be verified that $\alpha=0.99>\alpha^{*}$ and $\left(p^{N}, q^{N}\right) \in S$ for $N \in[3,8]$. The expected cost of compensation therefore increases over that range, but decreases outside that range.

The case where $N^{*} \leqslant N \leqslant \widetilde{N}$.

In this case, the limited liability constraint is binding (because $N \geqslant N^{*}$ ), but the degree of reporting flexibility is sufficiently low so that an increase in reporting

$\overline{10 \text { Remember that } \widetilde{N} \text { depends on }} \alpha, p$, and $q$, and that $\alpha^{*}$ depends on $\left(p^{N}, q^{N}\right)$. 

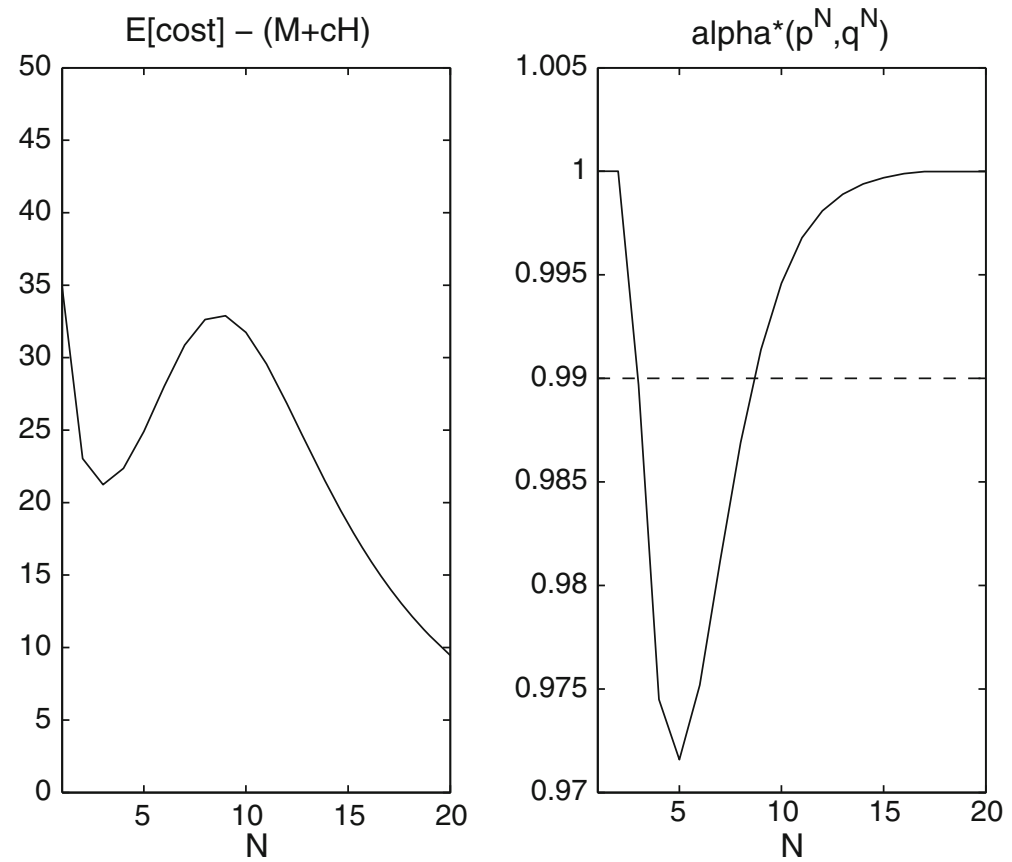

Fig. 4 Left panel expected cost of compensation in excess of $M+c_{H}$ as a function of $N$, for $c_{L}=0, c_{H}=$ $100, M=85000, p=0.46, q=0.52$, and $\alpha=0.99$. Right panel the critical value $\alpha^{*}$ as a function of $N$ for $p=0.46, q=0.52$

flexibility allows for a lower bonus (because $N \leqslant \widetilde{N}$ ). It then follows from Theorem 5 (ii) that the cost decreasing effects (decreased risk compensation and decreased incentive problems) dominate the limited liability effect if the manager is sufficiently risk averse, i.e. if $\alpha<\alpha_{b}^{*}$. Combined with the results of Theorem 3, this yields the following:

Implication 3: If $N^{*} \leqslant N \leqslant \widetilde{N}$ and $\alpha<\alpha_{b}^{*}$, an increase in the degree of reporting flexibility is strictly beneficial to both the manager and the principal.

We illustrate this result in Fig. 5.

For the parameter values in Fig. 5 , it can be verified that $N^{*}=2$, and $\widetilde{N}=26$, and that $\alpha=0.98<\alpha_{b}^{*}$ for all $N \leqslant 5$. Combined with Implication 1 , this implies that the expected cost of compensation decreases for $N \leqslant 5$, and increases for $N>5$. Interestingly, for values of $N$ between 2 and 5 , the expected cost of compensation is decreasing in $N$, even though an increase in $N$ strictly increases the manager's rent.

The case where $N \geqslant \max \left\{N^{*}, \widetilde{N}\right\}$.

In this case, the limited liability constraint is binding, and an increase in reporting flexibility would require a higher bonus. We know from Theorem 4 that the combination of increased incentive problems and limited liability then implies that the expected cost of compensation will increase when the level of reporting flexibility increases. Moreover, it follows from Theorem 3 that the manager's limited liability rent will also increase. 

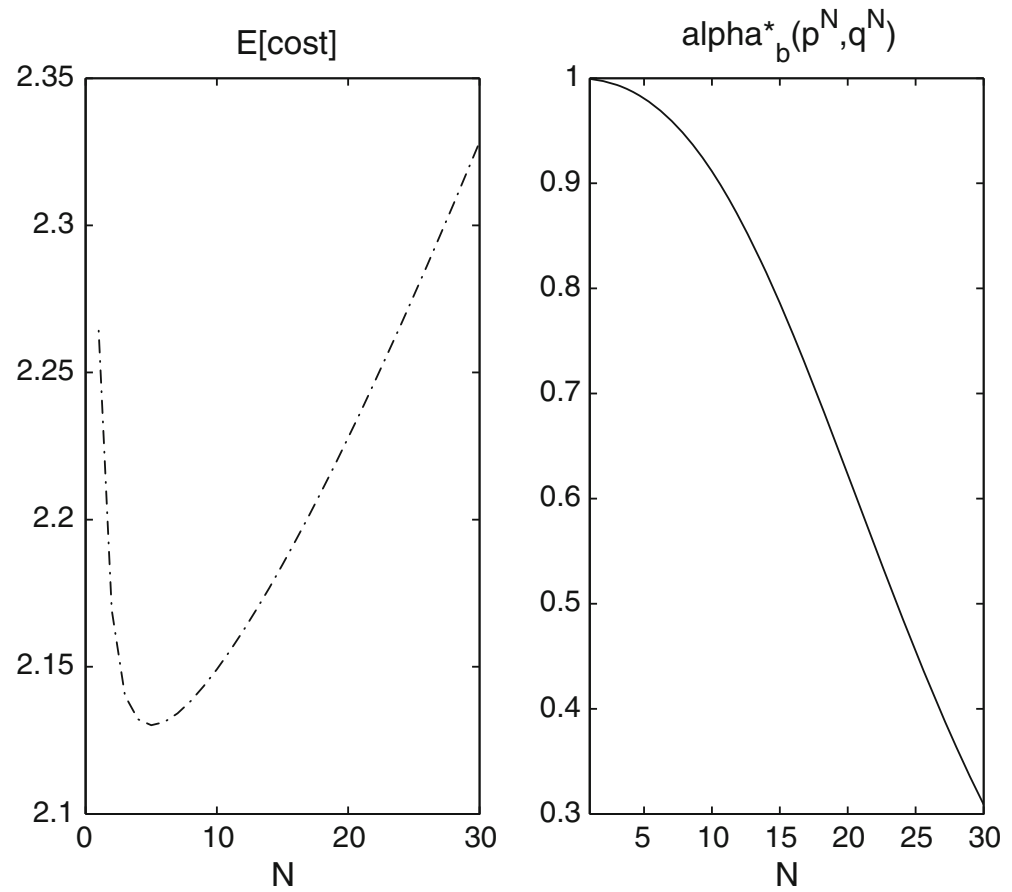

Fig. 5 The left panel the expected cost of compensation as a function of $N$, for $p=0.875, q=0.995$, $\rho=0.01, c_{L}=0, c_{H}=2$ and $M=0.094$, so that $\alpha=0.98$. The right panel $\alpha_{b}^{*}$ as a function of $N$, for $p=0.875$ and $q=0.995$

Implication 4: If $N$ is sufficiently high $\left(N \geqslant \max \left\{N^{*}, \tilde{N}\right\}\right)$, an increase in the degree of reporting flexibility makes the principal strictly worse off, while making the manager strictly better off.

\section{The effect of costly reporting effort}

Determining performance signals from multiple measurement methods may require costly effort on the part of the manager. In this section we investigate the effect of such flexibility induced reporting costs on the expected cost of compensation, and on the optimal degree of reporting flexibility.

Flexibility induced reporting costs affect incentive contracting in several ways.

(i) First, in determining the optimal levels of pay conditional on a given degree of reporting flexibility $N$, the principal needs to take into account that the degree of flexibility not only affects the probability distribution of the reported signal, but also the manager's disutility for reporting effort.

(ii) Second, when determining performance signals from different methods requires costly effort, the manager may not always have an incentive to determine all signals. Specifically, when $N$ methods are allowed, the manager will stop determining signals as soon as a high signal is found. As long as only low signals 
were found, the manager trades off the increase in the expected utility from compensation when more signals are determined against the higher disutility from searching effort. Whether it is beneficial to determine an additional signal depends on the bonus received in case a high signal is found, the probability that a high signal is found (which, in turn, depends on the action $a$ that was chosen), and the incremental cost for determining another signal. We consider the case where the monetary equivalent of the manager's disutility associated with determining the signals corresponding to $k \leqslant N$ methods is given by $\gamma(k)$, where $\gamma(k)$ is strictly increasing in $k$. Because the manager always stops evaluating signals once a favorable signal is found, his strategy is fully characterized by how long he continues to search for a favorable signal. We denote $\bar{n} \in\{1, \ldots, N\}$ for the manager's choice regarding the number of consecutive low signals after which he stops determining signals. Moreover, we denote

$$
N_{H}^{*}(s, N) \text { and } N_{L}^{*}(s, N),
$$

for the manager's optimal choice of $\bar{n}$ as a function of contract payments $s=$ $\left(s_{H}, s_{L}\right)$ and number of acceptable methods $N$, given that high or low action was chosen, respectively. A formal derivation of $N_{H}^{*}(s, N)$ and $N_{L}^{*}(s, N)$ is given in Lemma 1 in the Appendix.

(iii) Because the manager stops determining signals when either a high signal is found, or when the first $\bar{n}$ methods yielded low signals, the monetary equivalent of the disutility for effort spent on determining signals, denoted as $\widehat{\gamma}$, depends on the realizations of the signals. The joint probability distribution of the monetary equivalent of the disutility for reporting effort, $\widehat{\gamma}$, and the reported signal, $\widehat{y}$, depends on the manager's reporting choice $\bar{n}$, which determines the maximum number of signals that will be determined, and his action choice $a$, which determines the probability that a high signal is found before the maximum number of signals is determined. First consider the case where the manager chooses high action and evaluates at most $\bar{n}$ methods. Then, for any $k=1, \ldots, \bar{n}$, it holds that the manager will evaluate $k$ methods and report a high signal (i.e., $\widehat{\gamma}=\gamma(k)$, and $\widehat{y}=y_{H}$ ) iff the first $k-1$ signals are low and the $k$-th signal is high. This occurs with probability $(1-p) p^{k-1}$. He will evaluate $k=\bar{n}$ methods and report a low signal (i.e., $\widehat{\gamma}=\gamma(\bar{n})$, and $\widehat{y}=y_{L}$ ) iff the first $\bar{n}$ signals are low. This occurs with probability $p^{\bar{n}}$. Thus,

$$
\begin{aligned}
P\left(\widehat{\gamma}=\gamma(k), \widehat{y}=y_{H} \mid a=a_{H}, \bar{n}\right) & =(1-p) p^{k-1} & & \text { for } k=1, \ldots, \bar{n} . \\
P\left(\widehat{\gamma}=\gamma(k), \widehat{y}=y_{L} \mid a=a_{H}, \bar{n}\right) & =0 & & \text { for } k=1, \ldots, \bar{n}-1, \\
& =p^{k}, & & \text { for } k=\bar{n} .
\end{aligned}
$$

In case of low action, the probability distribution is as in (25) with $p$ replaced by $q$.

For any given degree of flexibility $N$, the principal determines contract payments $s_{H}=s\left(y_{H}, N\right)$ and $s_{L}=s\left(y_{L}, N\right)$ so as to minimize the expected cost of inducing the agent to choose action $a_{H}$, taking into account his self-interested behavior with 
respect to his action and reporting choices. For the latter, the principal needs to take into account that the contract payments affect the maximum number of signals that the manager will determine when high (low) action was chosen, i.e., they affect $N_{H}^{*}(s, N)$ and $N_{L}^{*}(s, N)$. This yields the following optimization problem:

$$
\begin{array}{ll}
\min _{s} & \mathbb{E}\left[s(\widehat{y}, N) \mid a=a_{H}, \bar{n}=N_{H}^{*}(s, N)\right] \\
& \widehat{y} \in \arg \max _{y \in\left\{y^{i}: i=1, \ldots, N_{H}^{*}(s, N)\right\}} s(y, N) \\
\text { s.t. } & \mathbb{E}\left[-e^{-\rho\left(s(\widehat{y}, N)-c_{H}-\widehat{\gamma}\right)} \mid a=a_{H}, \bar{n}=N_{H}^{*}(s, N)\right] \geqslant \bar{M} \\
& \mathbb{E}\left[-e^{-\rho\left(s(\widehat{y}, N)-c_{H}-\widehat{\gamma}\right)} \mid a=a_{H}, \bar{n}=N_{H}^{*}(s, N)\right] \\
& \geqslant \mathbb{E}\left[-e^{\left.-\rho\left(s(\widehat{y}, N)-c_{L}-\widehat{\gamma}\right)\right)} \mid a=a_{L}, \bar{n}=N_{L}^{*}(s, N)\right] \\
& s\left(y_{H}, N\right), s\left(y_{L}, N\right) \geqslant 0 .
\end{array}
$$

The (IC') constraint reflects the manager's self-interested behavior with respect to his reporting strategy, i.e., he will report the best signal out of maximum $N_{H}^{*}(s, N)$ signals, where $N_{H}^{*}(s, N)$ depends on the contractually agreed payments $\left(s\left(y_{H}, N\right), s\left(y_{L}, N\right)\right)$. The (IC) and (IR) constraints reflect the fact that accepting the contract and choosing high action must be optimal, taking into account that the action choice affects the probability distribution of both the the disutility for reporting effort $\widehat{\gamma}$, and the reported signal $\widehat{y}$.

The following theorem shows that if reporting costs are not too high, it is optimal for the principal to offer a contract that provides the manager with the incentive to keep searching for a favorable signal ultimately until all $N$ acceptable methods are used.

Theorem 7 The optimal contract satisfies $N_{H}^{*}(s, N)=N_{L}^{*}(s, N)=N$ if

$$
\max _{k \in\{1, \ldots, N-1\}}\left\{e^{\rho(\gamma(k+1)-\gamma(k))}\right\} \leqslant \frac{1}{(1-q) \cdot e^{-\rho \cdot B(i, j)}+q}, \quad \text { for all } i, j \leqslant N,
$$

where $B(i, j)$ denotes the the minimal required bonus to motivate the manager to choose high action, given that $\bar{n}=i$ if $a=a_{H}$ and $\bar{n}=j$ if $a=a_{L}$.

If condition (27) is satisfied, there does not exist a contract that satisfies the following two conditions. First, it motivates the manager to choose high action. Second, for at least one action choice it holds that once effort has been delivered and performance signals have been realized, the contract motivates the manager to stop searching for a favorable signal before all allowed methods are investigated (i.e., $N_{H}^{*}(s, N)<N$ and/or $\left.N_{L}^{*}(s, N)<N\right)$. The reason that such a contract does not exist if condition (27) is satisfied is that while the bonus offered in case of a favorable signal needs to be sufficiently high to motivate the agent to deliver high action, the bonus needs to be sufficiently low to provide incentives to stop searching for a favorable signal before all acceptable methods have been used. Condition (27) implies that whenever the bonus is sufficiently high to motivate high action, it will also induce the manager to keep searching for a favorable signal ultimately until all methods have been used. Consequently, any contract that motivates the agent to deliver high action necessarily satisfies $N_{H}^{*}(s, N)=N$ and $N_{L}^{*}(s, N)=N$. 


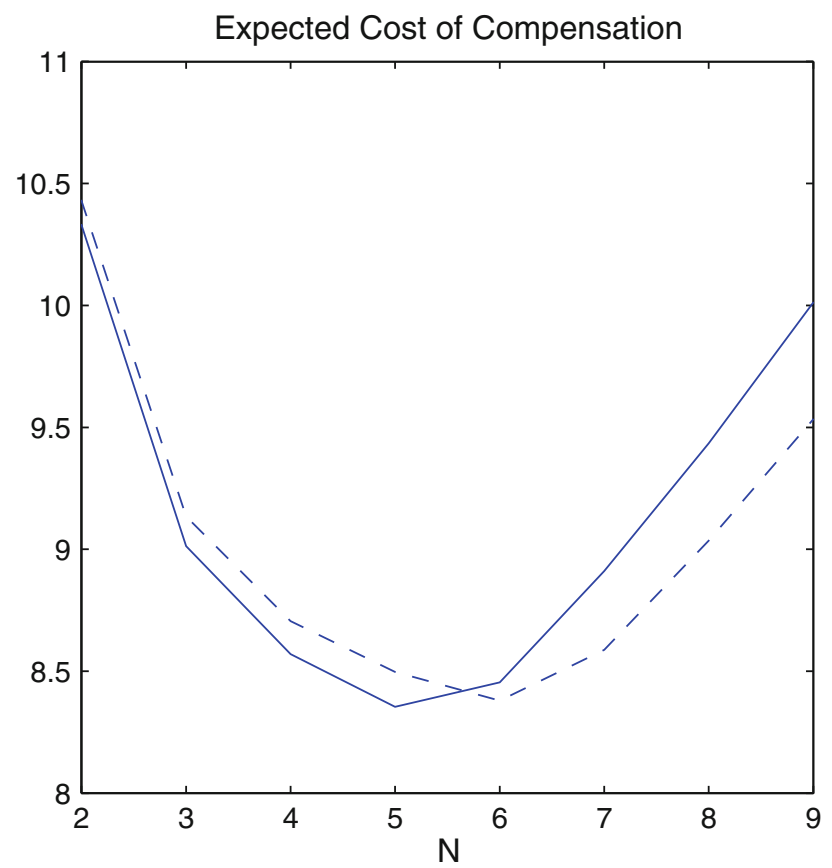

Fig. 6 Expected cost of compensation as a function of $N$ for $\rho=0.1, c_{L}=0, c_{H}=5, M=3, p=0.6$, $q=0.9$, and for $c=0$ (solid line), and $c=c_{H} / 80$ (dashed line), respectively

In the remainder of this section we show that flexibility induced costly reporting effort may make higher degrees of flexibility, i.e., a higher value of $N$, more attractive for the principal. Moreover, for sufficiently large degrees of reporting flexibility, the expected cost of compensation with costly reporting effort can be strictly less than the expected cost of compensation with costless reporting effort. Compared to the case where reporting effort is costless, the level of compensation may need to be increased for the effort-averse manager to (weakly) prefer the contract to his best outside option. However, when the contract induces the manager to engage in more costly reporting effort when low action was chosen than when high action was chosen, reporting costs make it easier to motivate the manager to choose high action, so that a lower bonus is needed. This suggests that higher degrees of flexibility can be more attractive when reporting induces costly effort than when reporting effort is costless. This is illustrated in Fig. 6.

Figure 6 displays the expected cost of compensation as a function of the number of acceptable measurement methods $N$ over the range $N \in[2,9]$, for the case where determining signals is costless, i.e., $\gamma(k)=0$, for all $k \leqslant N$ (solid line), and for the case where it induces costly reporting effort. In the latter case, the manager's monetary equivalent of the disutility associated with determining signals increases linearly in the number of signals that are determined, i.e., $\gamma(k)=c k$, with $c=c_{H} / 80$ (dashed line).

Both with costless and with costly reporting effort, a minimum of two methods is needed for an optimal contract to exist, but allowing more reporting flexibility is optimal for the principal; the expected cost of compensation decreases significantly as $N$ 

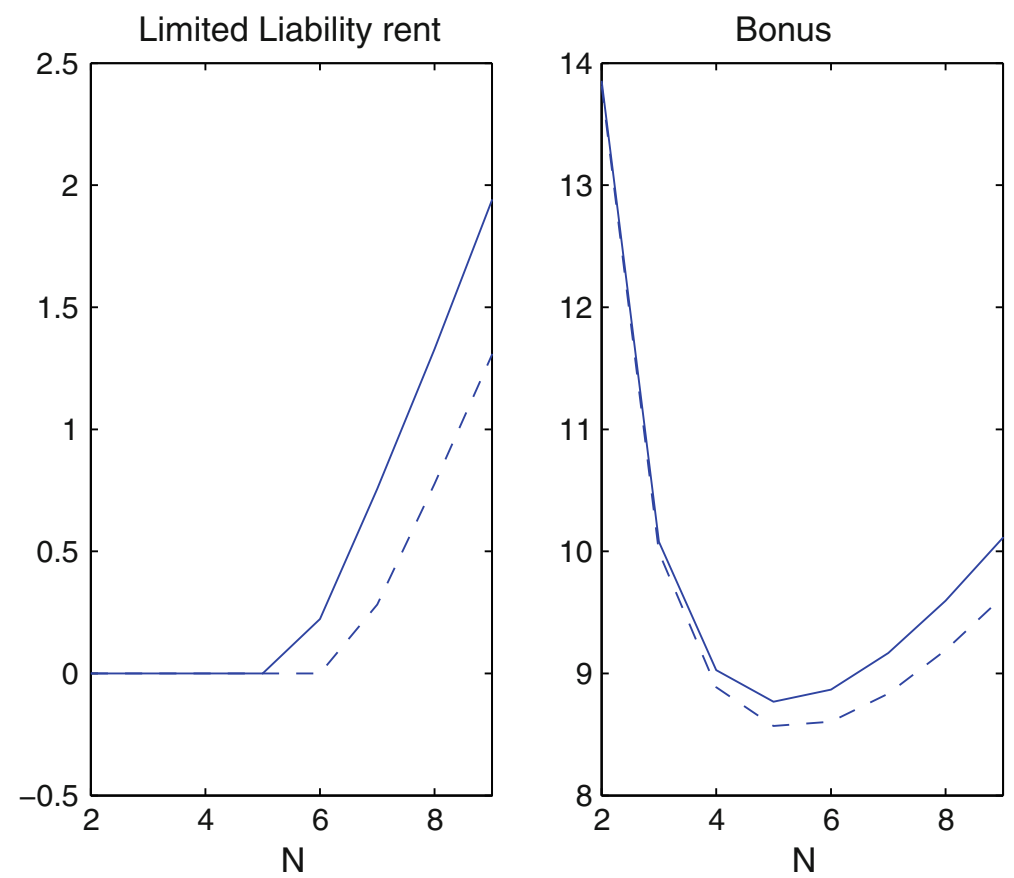

Fig. 7 The limited liability rent (left panel) and the bonus (right panel) as a function of $N$ for $\rho=0.1$, $c_{L}=0, c_{H}=5, M=3, p=0.6, q=0.9$, and for $c=0$ (solid line), and $c=c_{H} / 80$ (dashed line), respectively

increases from $N=2$ to $N=5$. However, the optimal degree of reporting flexibility when reporting is costly is higher than when it is costless. Indeed, whereas it is optimal for the principal to allow the manager to choose out of maximum $N=5$ methods in case reporting is costless, the optimal level of reporting flexibility increases to $N=6$ when it is costly. Moreover, for any $N>5$, the expected cost of compensation with costly reporting effort is strictly lower than the expected cost of compensation with costless reporting effort. This can be explained by the following two reasons.

First, it can be verified that for all $N$, condition (27) is satisfied. Thus, regardless of whether high or low action was chosen, the manager will continue to evaluate signals until either a favorable signal is found, or all $N$ methods were used. The probability of finding a favorable performance signal, however, is higher when high action was chosen than when low action was chosen. Therefore, the manager will, in expectation, engage in more costly reporting effort in an attempt to increase his compensation when low action was chosen. This implies that high action becomes relatively more attractive when reporting requires costly effort, so that a lower bonus suffices to motivate the manager to choose high action. This is illustrated in the right panel in Fig. 7.

Second, the analysis in the previous sections shows that the effect of reporting flexibility on the expected cost of compensation depends also crucially on how it affects the manager's limited liability rent. When the degree of reporting flexibility is suffi- 
ciently high (i.e., when $N>N^{*}=5$ ), the manager would earn a limited liability rent when reporting effort would be costless. In contrast, when determining performance signals induces costly effort, the increase in the level of pay needed to compensate the effort-averse manager for this effort implies that the limited liability constraint is only binding when $N>6$. Moreover, for any $N>5$, costly reporting effort strictly reduces the manager's limited liability rent. This is illustrated in the left panel in Fig. 7. Thus, a higher degree of reporting flexibility is more attractive when reporting effort is costly than when it is costless, because the higher level of pay needed to compensate the manager for the disutility associated with reporting effort is fully outweighed by the decrease in both the bonus and the limited liability rent.

\section{Conclusion}

This paper studies the effect of the degree of reporting flexibility on agency costs in settings where the principal delegates measurement method choice to the agent.

First, we show that a minimal degree of reporting flexibility may be necessary to be able to resolve incentive conflicts at finite cost. If the principal faces a hard agency problem because an unfavorable signal carries little information regarding the manager's action choice and because the level of utility that the manager derives from compensation cannot be made arbitrarily high by increasing the level of the bonus in case of a favorable signal, it is impossible to design a contract that motivates the manager to deliver high effort. Increasing the degree of reporting flexibility increases the informativeness of an unfavorable signal. Therefore, if a manager cannot be motivated to deliver high effort by increasing the level of the bonus, increasing the level of reporting discretion may be an alternative means to resolve incentive conflicts.

Second, we identify the three main effects of an increase in the level of reporting flexibility on managerial compensation in a setting where the manager has the discretion to choose the method. First, it reduces the manager's risk because the probability that he will be able to report a favorable signal increases. Second, the size of the bonus required to motivate the manager to provide high effort can be decreased if the current level of discretion is sufficiently low, but the opposite would happen if that level is relatively high. Finally, the fact that the manager faces limited liability significantly affects the effect of increased reporting flexibility. The limited liability constraint will be binding if the degree of reporting flexibility is, or becomes, sufficiently high. We characterize the conditions under which the aggregate of these three effects will lead to a higher or lower cost of compensation. We find that for a broad set of parameter values, increased reporting flexibility would be strictly beneficial to the principal and would leave the manager's utility unaffected. We also identify conditions under which both the principal and the manager are strictly better off when more performance measurement alternatives are available.

Finally, we investigate the effect of costly reporting effort on the expected compensation cost. We find that, compared to the case where reporting effort is costless, offering more reporting flexibility may be more attractive. Moreover, for high degrees of flexibility, the expected cost of compensation can be strictly lower when reporting effort is costly than when it is costless. This occurs for two reasons. First, when 
search costs are not too high, the manager has an incentive to continue to evaluate performance signals until either a favorable signal is found or all acceptable methods were used. Because the probability of finding a favorable performance signal is higher when high action was chosen than when low action was chosen, the manager will, in expectation, engage in more costly reporting effort when low action was chosen. Thus, costs associated with determining performance signals make the low action choice relatively less attractive, so that a lower bonus suffices to motivate the agent to choose high action. Second, when the degree of reporting flexibility is sufficiently high, compensation for costly reporting effort may lead to a reduction in the limited liability rent. The combination of these two effects implies that higher degrees of flexibility can be more attractive with costly reporting effort than with costless reporting effort.

Acknowledgments We wish to thank two anonymous reviewers and Michael Kirschenheiter for comments that helped to improve the paper significantly.

Open Access This article is distributed under the terms of the Creative Commons Attribution Noncommercial License which permits any noncommercial use, distribution, and reproduction in any medium, provided the original author(s) and source are credited.

\section{Appendix A: Proofs}

Remarks 1. Note that if a real-valued function $f(\cdot)$ is increasing (decreasing) over the range $[l, b]$, then $f(\cdot)$ is clearly also increasing (decreasing) over all integer values in that range. Therefore, although $N$ can only take integer values, we can conclude that $f(N)$ is increasing (decreasing) in $N$ over a certain range if $f^{\prime}(\cdot)>0(<0)$ over that range.

2. The following properties will be used throughout the proofs:

$$
\begin{array}{ll}
\frac{d}{d n}\left(x^{n}\right)=x^{n} \ln (x), & \text { for all } x>0, \\
\ln (x)<0, & \text { for all } x \in[0,1], \\
\ln (x) \leqslant x-1, & \text { for all } x>0, \\
\ln \left(x^{n}\right)=n \ln (x), & \text { for all } x>0 \text { and } n \in \mathbb{N}, \\
\ln (x y)=\ln (x)+\ln (y), & \text { for all } x>0, y>0 .
\end{array}
$$

Proof of Theorem 1 (i) Let us first consider the optimization problem without the limited liability constraints. Then it follows immediately from the KKT-conditions that the individual rationality and the incentive compatibility constraint are both binding.

It can be verified that the solution equals $s_{L}(N)=-\frac{1}{\rho} \ln \left(x^{*}\right)$ and $s_{H}(N)=$ $-\frac{1}{\rho} \ln \left(y^{*}\right)$, where

$$
\begin{aligned}
x^{*} & =\frac{\left(1-p^{N}\right) e^{-\rho\left(M+c_{L}\right)}-\left(1-q^{N}\right) e^{-\rho\left(M+c_{H}\right)}}{q^{N}-p^{N}}, \\
y^{*} & =\frac{q^{N} e^{-\rho\left(M+c_{H}\right)}-p^{N} e^{-\rho\left(M+c_{L}\right)}}{q^{N}-p^{N}} .
\end{aligned}
$$


The resulting payment scheme is feasible (i.e., $0 \leqslant s_{L}<\infty, 0 \leqslant s_{H}<\infty$ ) iff $0<x^{*} \leqslant 1$ and $0<y^{*} \leqslant 1$. If $x^{*} \leqslant 0$ or $y^{*} \leqslant 0$, then an optimal compensation scheme does not exist. If $x^{*}>1$ or $y^{*}>1$, then the limited liability constraint is violated.

Note that $y^{*} \leqslant x^{*}$ and

$$
y^{*}>0 \Longleftrightarrow\left(\frac{q}{p}\right)^{N}>e^{\rho\left(c_{H}-c_{L}\right)},
$$

so that indeed an optimum exists iff $N>\frac{\rho\left(c_{H}-c_{L}\right)}{\ln q-\ln p}$.

Furthermore, the limited liability constraint is binding iff

$$
x^{*}>1 \Longleftrightarrow \frac{1-q^{N}}{1-p^{N}}>\frac{1-e^{-\rho\left(M+c_{L}\right)}}{1-e^{-\rho\left(M+c_{H}\right)}} .
$$

Now it remains to show that

$$
N>N^{*} \Longleftrightarrow \frac{1-q^{N}}{1-p^{N}}>\frac{1-e^{-\rho\left(M+c_{L}\right)}}{1-e^{-\rho\left(M+c_{H}\right)}} .
$$

Given the definition of $N^{*}$, it is sufficient to show that $\frac{1-q^{N}}{1-p^{N}}$ is increasing in $N$.

$$
\begin{aligned}
\frac{d}{d N}\left(\frac{1-q^{N}}{1-p^{N}}\right) & =\frac{p^{N} \ln p\left(1-q^{N}\right)-q^{N} \ln q\left(1-p^{N}\right)}{\left(1-p^{N}\right)^{2}} \\
& =\frac{1-q^{N}}{1-p^{N}}\left(\frac{p^{N}}{1-p^{N}} \ln p-\frac{q^{N}}{1-q^{N}} \ln q\right)
\end{aligned}
$$

Now, let us introduce the function

$$
g(x)=\frac{x \ln x}{1-x}
$$

Then

$$
\begin{aligned}
g^{\prime}(x) & =\frac{(\ln x+1)(1-x)+x \ln x}{(1-x)^{2}} \\
& =\frac{\ln x-x \ln x+1-x+x \ln x}{(1-x)^{2}}, \\
& =\frac{\ln x+1-x}{(1-x)^{2}} \leqslant 0
\end{aligned}
$$


The last inequality follows from the fact that $\ln x \leqslant x-1$. Therefore,

$$
\begin{aligned}
\frac{d}{d N}\left(\frac{1-q^{N}}{1-p^{N}}\right) & =\frac{1-q^{N}}{1-p^{N}}\left(g\left(p^{N}\right)-g\left(q^{N}\right)\right) \cdot \frac{1}{N}, \\
& \geqslant 0 .
\end{aligned}
$$

Moreover,

$$
\left(\frac{1-q^{N}}{1-p^{N}}\right)_{N=1}=\frac{1-q}{1-p}<1
$$

and

$$
\lim _{N \rightarrow \infty} \frac{1-q^{N}}{1-p^{N}}=1
$$

Therefore,

$$
\frac{1-q^{N}}{1-p^{N}} \leqslant \frac{1-e^{-\rho\left(M+c_{L}\right)}}{1-e^{-\rho\left(M+c_{H}\right)}} \Leftrightarrow N \leqslant N^{*} .
$$

(ii) It follows from the proof of (i) that

$$
\frac{1-q^{N}}{1-p^{N}}>\frac{1-e^{-\rho\left(M+c_{L}\right)}}{1-e^{-\rho\left(M+c_{H}\right)}}
$$

implies that $x^{*}>1$, so that the limited liability constraint $s_{L}(N) \geqslant 0$ is binding. It then follows that the optimal compensation under $y_{H}$ satisfies

$$
\begin{array}{ll}
s_{H}(N)=\min -\frac{1}{\rho} \ln (y) \\
\text { s.t. } & e^{\rho c_{H}}\left[p^{N} x+\left(1-p^{N}\right) y\right] \leqslant e^{\rho c_{L}}\left[q^{N} x+\left(1-q^{N}\right) y\right] \\
& e^{\rho c_{H}}\left[p^{N} x+\left(1-p^{N}\right) y\right] \leqslant e^{-\rho M} \\
& 0<y \leqslant 1 \\
& x=1
\end{array}
$$

It can be verified that the incentive compatibility constraint is binding, ${ }^{11}$ and

$$
s_{H}(N)=-\frac{1}{\rho} \ln \left(y_{c}^{*}\right),
$$

\footnotetext{
${ }^{11}$ Let $y_{c}^{*}$ denote the optimum of (32), and let $x^{*}$ and $y^{*}$ be as given in (28) and (29). It can be verified that the IC constraint is equivalent to $y \leq y^{*} / x^{*}$, and that the IR constraint is equivalent to $y \leq y_{r}^{*}=$ $\left(e^{-\rho\left(M+c_{H}\right)}-p^{N}\right) /\left(1-p^{N}\right)$. In the optimum, at least one constraint is binding, because otherwise $y$ can be increased. Therefore, $y_{c}^{*}=\min \left\{y^{*} / x^{*}, y_{r}^{*}\right\}$. By construction, $x^{*}$ and $y^{*}$ satisfy both the IC and the IR constraint in equality, and $x^{*}>1$. This implies that for $(x, y)=\left(x^{*} / x^{*}, y^{*} / x^{*}\right)$, the IC constraint is binding and the IR constraint is slack, which implies that $y_{c}^{*}=y^{*} / x^{*} \leq y_{r}^{*}$.
} 
where

$$
y_{c}^{*}=\frac{q^{N} e^{-\rho\left(M+c_{H}\right)}-p^{N} e^{-\rho\left(M+c_{L}\right)}}{\left(1-p^{N}\right) e^{-\rho\left(M+c_{L}\right)}-\left(1-q^{N}\right) e^{-\rho\left(M+c_{H}\right)}} .
$$

Proof of Theorem 2 Let us introduce the following notation:

$$
\begin{aligned}
\tilde{x} & =\frac{q^{N} \alpha-p^{N}+(1-\alpha)}{q^{N}-p^{N}}, \\
\tilde{y} & =\frac{q^{N} \alpha-p^{N}}{q^{N}-p^{N}}, \\
\tilde{y}_{c} & =\frac{q^{N} \alpha-p^{N}}{q^{N} \alpha-p^{N}+(1-\alpha)} .
\end{aligned}
$$

Then, for $N \leqslant N^{*}$,

$$
\begin{aligned}
& s_{L}(N)=M+c_{L}-\frac{1}{\rho} \ln (\widetilde{x}), \\
& s_{H}(N)=M+c_{L}-\frac{1}{\rho} \ln (\widetilde{y}),
\end{aligned}
$$

and for $N>N^{*}$

$$
s_{H}(N)=-\frac{1}{\rho} \ln \left(\widetilde{y_{c}}\right) .
$$

Moreover, for all $N$,

$$
s_{H}(N)-s_{L}(N)=-\frac{1}{\rho} \ln \tilde{y}_{c} .
$$

Therefore, it is sufficient to show that: i) $\tilde{x}$ is increasing in $N$, ii) $\tilde{y}$ is increasing in $N$, and iii) $\widetilde{y_{c}}$ is increasing in $N$ for $N \leqslant \widetilde{N}$, and decreasing in $N$ for $N>\widetilde{N}$.

(i)

$$
\begin{aligned}
\frac{d \widetilde{x}}{d N}= & \frac{\left(q^{N} \cdot \ln q \cdot \alpha-p^{N} \cdot \ln p\right) *\left(q^{N}-p^{N}\right)}{\left(q^{N}-p^{N}\right)^{2}} \\
& -\frac{\left(q^{N} \cdot \ln q-p^{N} \cdot \ln p\right) *\left(q^{N} \alpha-p^{N}\right)}{\left(q^{N}-p^{N}\right)^{2}} \\
= & \frac{1-\alpha}{\left(q^{N}-p^{N}\right)^{2}} \cdot\left(\ln p \cdot p^{N}\left(1-q^{N}\right)-\ln q \cdot q^{N}\left(1-p^{N}\right)\right)
\end{aligned}
$$

Therefore,

$$
\frac{d \tilde{x}}{d N}=\frac{(1-\alpha)\left(1-p^{N}\right)\left(1-q^{N}\right)}{N\left(q^{N}-p^{N}\right)^{2}}\left(g\left(p^{N}\right)-g\left(q^{N}\right)\right) \geqslant 0,
$$

where the function $g(\cdot)$ is as defined in (31). 
(ii) Since

$$
\widetilde{y}=\widetilde{x}-\frac{1-\alpha}{q^{N}-p^{N}},
$$

it follows that

$$
\begin{aligned}
\frac{d \widetilde{y}}{d N}= & \frac{d \widetilde{x}}{d N}-(1-\alpha) \frac{d}{d N}\left(\frac{1}{q^{N}-p^{N}}\right) \\
= & \frac{(1-\alpha)}{\left(q^{N}-p^{N}\right)^{2}}\left[\ln p \cdot p^{N}\left(1-q^{N}\right)-\ln q \cdot q^{N}\left(1-p^{N}\right)-\ln p \cdot p^{N}\right. \\
& \left.+\ln q \cdot q^{N}\right], \\
= & \frac{(1-\alpha)}{\left(q^{N}-p^{N}\right)^{2}} p^{N} q^{N}[\ln q-\ln p] \\
\geqslant & 0 .
\end{aligned}
$$

(iii) It can be verified that

$$
\frac{d y_{c}^{*}}{d N}=(1-\alpha) \cdot \frac{\alpha q^{N} \ln q-p^{N} \ln p}{\left(\alpha q^{N}-p^{N}+1-\alpha\right)^{2}},
$$

which is negative iff

$$
\frac{\ln q}{\ln p}\left(\frac{q}{p}\right)^{N} \geqslant \frac{1}{\alpha} \Longleftrightarrow N \geqslant \tilde{N} .
$$

Proof of Theorem 3 Let us denote $\widetilde{s}_{L}(N)$ and $\widetilde{s}_{H}(N)$ for the optimal compensation scheme when there is no limited liability constraint. Then it follows immediately from the proof of Theorem 1 that $\widetilde{s}_{L}(N)$ and $\widetilde{s}_{H}(N)$ are given by (13) and (14), respectively, for all $N$. Moreover,

$$
\begin{array}{lll}
s_{L}(N)=\widetilde{s}_{L}(N), & s_{H}(N)=\widetilde{s}_{H}(N), & \text { if } N \leqslant N^{*}, \\
s_{L}(N)=\widetilde{s}_{L}(N)-\widetilde{s}_{L}(N)=0, & s_{H}(N)=\widetilde{s}_{H}(N)-\widetilde{s}_{L}(N), & \text { if } N>N^{*} .
\end{array}
$$

i.e., as a consequence of the limited liability constraint, the compensation increases with the amount $-\widetilde{s}_{L}(N)$.

As can be seen from the proof of Theorem 1, the individual rationality constraint is binding when $N<N^{*}$. Given (37), this implies that the manager's certain equivalent is given by:

$$
\begin{aligned}
C E(N) & =M+c_{H}, & & \text { if } N \leqslant N^{*}, \\
& =M+c_{H}-\widetilde{s}_{L}(N), & & \text { if } N>N^{*} .
\end{aligned}
$$


The limited liability rent, $C E(N)-\left(M+c_{H}\right)$, is zero when $N \leqslant N^{*}$, and equals $-\widetilde{s}_{L}(N)$ when $N>N^{*}$. It follows from the proof of Theorem 2 that $-\widetilde{s}_{L}(N)$ is strictly increasing in $N$.

Proof of Theorem 4 Suppose that $N>\max \left\{N^{*}, \widetilde{N}\right\}$. Then, we know that $s_{L}(N)=0$ and $s_{H}(N)$ is increasing in $N$. Moreover, since $s_{L}(N)=0$, the expected cost equals

$$
\left(1-p^{N}\right) \cdot s_{H}(N)
$$

The fact that $1-p^{N}$ is increasing in $N$ completes the proof.

Proof of Theorem 5 First consider the case where $N \leqslant N^{*}$. Then the derivative with respect to $N$ of the expected cost equals

$$
D(\alpha)=-p^{N} \ln p\left(s_{H}(N)-s_{L}(N)\right)+\left(1-p^{N}\right) \frac{d}{d N} s_{H}(N)+p^{N} \frac{d}{d N} s_{L}(N) .
$$

It follows from the proof of Theorem 2 that

$$
\begin{aligned}
\frac{d}{d N} s_{L}(N) & =-\frac{1}{\rho} \frac{1-\alpha}{\left(q^{N}-p^{N}\right)} \frac{\ln p \cdot p^{N}\left(1-q^{N}\right)-\ln q \cdot q^{N}\left(1-p^{N}\right)}{\left(1-p^{N}\right)-\left(1-q^{N}\right) \alpha} \\
\frac{d}{d N} s_{H}(N) & =-\frac{1}{\rho} \frac{1-\alpha}{\left(q^{N}-p^{N}\right)} \frac{p^{N} q^{N}(\ln q-\ln p)}{q^{N} \alpha-p^{N}}
\end{aligned}
$$

Some straightforward but tedious computations show that

$$
D(\alpha)=\frac{p^{N}}{\rho} G\left(\frac{q^{N} \alpha-p^{N}}{1-\alpha}\right)
$$

where

$$
\begin{aligned}
G(z)= & \frac{1}{z^{2}+z} \cdot \frac{\ln p \cdot p^{N}\left(1-q^{N}\right)-\ln q \cdot q^{N}\left(1-p^{N}\right)}{q^{N}-p^{N}} \\
& +\ln p\left(\frac{1}{z}-\ln \left(1+\frac{1}{z}\right)\right) .
\end{aligned}
$$


Our goal is to determine the sign of $D(\alpha)$. Now, first notice that

$$
\begin{aligned}
\lim _{z \rightarrow 0} G(z)= & \lim _{z \rightarrow 0} \frac{1}{z}\left(\frac{1}{1+z} \frac{\left(\ln p \cdot p^{N}\left(1-q^{N}\right)-\ln q \cdot q^{N}\left(1-p^{N}\right)\right)}{\left(q^{N}-p^{N}\right)}+\ln p\right. \\
& \left.-\ln p \frac{\ln \left(1+\frac{1}{z}\right)}{\frac{1}{z}}\right) \\
= & \frac{(\ln p-\ln q) q^{N}\left(1-p^{N}\right)}{q^{N}-p^{N}} \lim _{z \rightarrow 0} \frac{1}{z} \\
= & -\infty . \\
\lim _{z \rightarrow \infty} G(z)= & 0
\end{aligned}
$$

For ease of notation, we define

$$
\begin{aligned}
c_{1} & =\frac{\ln p \cdot p^{N}\left(1-q^{N}\right)-\ln q \cdot q^{N}\left(1-p^{N}\right)}{q^{N}-p^{N}}>0, \\
c_{2} & =-\ln p>0, \\
f(z) & =\frac{1}{z+z^{2}}, \\
g(z) & =\frac{1}{z}-\ln \left(1+\frac{1}{z}\right) .
\end{aligned}
$$

Then

$$
G(z)=c_{1} f(z)-c_{2} g(z) \text {. }
$$

It can easily be verified that

$$
\begin{aligned}
& f^{\prime}(z)=-\frac{1+2 z}{z^{2}(1+z)^{2}}, \\
& g^{\prime}(z)=-\frac{1}{z^{2}(1+z)} .
\end{aligned}
$$

Consequently,

$$
G^{\prime}(z)=\frac{1}{z^{2}(1+z)^{2}}\left(\left(c_{2}-2 c_{1}\right) z+c_{2}-c_{1}\right)
$$


Now, notice that

$$
\begin{aligned}
c_{1}-c_{2}= & \frac{1}{q^{N}-p^{N}} \cdot\left(\ln p \cdot p^{N}\left(1-q^{N}\right)-\ln q \cdot q^{N}\left(1-p^{N}\right)\right. \\
& \left.+\ln p\left(q^{N}-p^{N}\right)\right), \\
= & \frac{1}{q^{N}-p^{N}}(\ln p-\ln q) \cdot q^{N}\left(1-p^{N}\right)<0 .
\end{aligned}
$$

Now, we consider the following two situations:

$-2 c_{1} \leqslant c_{2}$

Then since $c_{1}-c_{2}<0$, it follows that $G^{\prime}(z)>0$ for all $z$. Combined with (39) and (40) this implies that $G(z) \leqslant 0$ for all $z$.

$-2 c_{1}>c_{2}$

Then, since $c_{1}-c_{2}<0$ and $2 c_{1}-c_{2}>0$ we know that $G^{\prime}(z)$ has exactly one strictly positive root. Therefore, $G^{\prime}(z) \geqslant 0$ for $z \leqslant \widetilde{z}$ and $G^{\prime}(z)<0$ for $z>\widetilde{z}$, where $\widetilde{z}$ denotes the unique positive root of $G^{\prime}(z)$. Again, combined with (39) and (40), this implies that $G(z)$ has a unique positive root $z^{*}$. This implies that $D(\alpha) \leqslant 0$ for $\alpha \leqslant \alpha^{*}$, and $D(\alpha)>0$ for $\alpha>\alpha^{*}$ where

$$
\alpha^{*}=\frac{z^{*}+p^{N}}{z^{*}+q^{N}}
$$

It now only remains to see that, since $\ln p=\frac{\ln p^{N}}{N}$ and $\ln q=\frac{\ln q^{N}}{N}$, it follows that $\left(p^{N}, q^{N}\right) \in S$ iff $2 c_{1}>c_{2}$.

Now, consider the case where $N>N^{*}$. Then, given (37), it follows that the derivative with respect to $N$ of the expected cost equals

$$
D_{b}(\alpha)=-p^{N} \ln p\left(s_{H}(N)-s_{L}(N)\right)+\left(1-p^{N}\right) \frac{d}{d N} s_{H}(N)+\left(p^{N}-1\right) \frac{d}{d N} s_{L}(N),
$$

where $s_{H}(N)$ and $s_{L}(N)$ are as defined in (14) and (13), respectively.

Therefore,

$$
D_{b}(\alpha)=\frac{1}{\rho} G_{b}\left(\frac{q^{N} \alpha-p^{N}}{1-\alpha}\right)
$$

where

$$
\begin{aligned}
G_{b}(z)= & \left(p^{N} \frac{1}{z^{2}+z}+\frac{1}{1+z}\right) * \frac{\ln p \cdot p^{N}\left(1-q^{N}\right)-\ln q \cdot q^{N}\left(1-p^{N}\right)}{q^{N}-p^{N}} \\
& +p^{N} \ln p\left(\frac{1}{z}-\ln \left(1+\frac{1}{z}\right)\right) .
\end{aligned}
$$


Again,

$$
\begin{aligned}
\lim _{z \rightarrow 0} G_{b}(z) & =-\infty \\
\lim _{z \rightarrow \infty} G_{b}(z) & =0 .
\end{aligned}
$$

It can easily be verified that

$$
G_{b}^{\prime}(z)=\frac{1}{z^{2}(1+z)^{2}}\left(-\frac{c_{1}}{p^{N}} z^{2}+\left(c_{2}-2 c_{1}\right) z+c_{2}-c_{1}\right)
$$

where

$$
\begin{aligned}
& c_{1}=p^{N} \frac{\ln p \cdot p^{N}\left(1-q^{N}\right)-\ln q \cdot q^{N}\left(1-p^{N}\right)}{q^{N}-p^{N}}>0 \\
& c_{2}=-p^{N} \ln p>0 .
\end{aligned}
$$

Since $G_{b}^{\prime}(0)=+\infty$ and $-\frac{c_{1}}{p^{N}}<0$, if follows that $G_{b}^{\prime}(z)$ has exactly one strictly positive root. This concludes the proof.

Proof of Theorem 6 Let us denote $\widetilde{s}_{L}(N)$ and $\widetilde{s}_{H}(N)$ for the optimal compensation scheme when there is no limited liability constraint. Then it follows immediately from the proof of Theorem 1 that $\widetilde{s}_{L}(N)$ and $\widetilde{s}_{H}(N)$ are given by (13) and (14), respectively, for all $N$.

It follows from (37), and (38) that for all $N$, the risk premium is given by:

$$
\begin{aligned}
r p(N) & =p^{N} \cdot s_{L}(N)+\left(1-p^{N}\right) \cdot s_{H}(N)-C E(N) \\
& =p^{N} \cdot \widetilde{s}_{L}(N)+\left(1-p^{N}\right) \cdot \widetilde{s}_{H}(N)-\left(M+c_{H}\right) .
\end{aligned}
$$

The result now follows immediately from the proof of Theorem 5 for the case where $N \leqslant N^{*}$.

In order to be able to prove Theorem 7 , we first formulate the following lemma.

Lemma 1 For any given contract payments $s=\left(s_{L}, s_{H}\right)$ and number of acceptable methods $N$, the manager's optimal strategy when high action was chosen is to stop determining signals as soon as either a high signal is found, or $N_{H}^{*}(s, N)$ methods have been evaluated, where:

$$
N_{H}^{*}(s, N)=\min \left\{k: V(k)=\widetilde{u}\left(s_{L}, c_{H}+\gamma(k)\right)\right\},
$$

where $V(k)$ is determined recursively from: 


$$
\begin{aligned}
& V(k)=\widetilde{u}\left(s_{L}, c_{H}+\gamma(k)\right), \\
& \text { for } k=N \text {, } \\
& =\max \left\{\begin{array}{l}
\tilde{u}\left(s_{L}, c_{H}+\gamma(k)\right), \\
(1-p) \cdot \widetilde{u}\left(s_{H}, c_{H}+\gamma(k+1)\right)+p \cdot V(k+1)
\end{array}\right\}, \quad \text { for } k=N-1, \ldots, 1 .
\end{aligned}
$$

and $\widetilde{u}(x, c)=-e^{-\rho(x-c)}$ for all $x, c \in \mathbb{R}$.

The manager's optimal reporting strategy when low action was chosen is to stop determining signals as soon as either a high signal is found, or $N_{L}^{*}(s, N)$ methods have been evaluated, where $N_{L}^{*}(s, N)$ follows from (43) and (44) with p replaced by $q$, and $c_{H}$ by $c_{L}$.

Proof of lemma 1 First consider the case where $a=a_{H}$. Let $V(k)$ denote the maximal expected utility from compensation, net of the disutility for effort, given that the first $k$ signals were low. To decide whether one more signal is determined, the manager compares the utility in case he stops, i.e., $\widetilde{u}\left(s_{L}, c_{H}+\gamma(k)\right)$, with the maximal expected utility in case he determines at least one more signal. Determining one more signal implies that with probability $1-p$, a high signal is found. The manager's utility is then $\widetilde{u}\left(s_{H}, c_{H}+\gamma(k+1)\right)$, and it is optimal to stop searching. With probability $p$, a low signal is found, and the optimal expected utility is given by $V(k+1)$. Thus, the optimal expected utility in case one more signal is determined equals:

$$
(1-p) \cdot \widetilde{u}\left(s_{H}, c_{H}+\gamma(k+1)\right)+p \cdot V(k+1) .
$$

It is optimal to stop verifying signals as soon as

$$
\widetilde{u}\left(s_{L}, c_{H}+\gamma(k)\right) \geqslant(1-p) \cdot \widetilde{u}\left(s_{H}, c_{H}+\gamma(k+1)\right)+p \cdot V(k+1) .
$$

This implies that:

$$
N_{H}^{*}(s, N)=\min \left\{k: V(k)=\widetilde{u}\left(s_{L}, c_{H}+\gamma(k)\right)\right\}
$$

The proof for $a=a_{L}$ is similar.

Proof of Theorem 7 We first show that $N_{H}^{*}(s, N)$ and $N_{H}^{*}(s, N)$ are increasing in $s_{H}-s_{L}$. Consider the case where $a=a_{H}$. Let us denote $V\left(s_{L}, s_{H}, k\right)$ for the value function defined in Lemma 1 , as a function of the contract payments $s_{L}$ and $s_{H}$. It is verified easily that

$$
V\left(s_{L}, s_{H}, k\right)=e^{-\rho s_{L}} \cdot V\left(0, s_{H}-s_{L}, k\right)
$$

and that $V\left(0, s_{H}-s_{L}, k\right)$ is increasing in $s_{H}-s_{L}$. Moreover, stopping is optimal iff $\widetilde{u}\left(0, c_{H}+\gamma(k)\right) \geqslant(1-p) \cdot \widetilde{u}\left(s_{H}-s_{L}, c_{H}+\gamma(k+1)\right)+p \cdot V\left(0, s_{H}-s_{L}, k+1\right)$,

Because both $\widetilde{u}\left(s_{H}-s_{L}, c_{H}+\gamma(k+1)\right)$ and $V\left(0, s_{H}-s_{L}, k+1\right)$ are increasing in $s_{H}-s_{L}$, this implies that $N_{H}^{*}(s, N)$ is increasing in $s_{H}-s_{L}$. The proof for $a=a_{L}$ is similar. 
Next, we determine a sufficient condition for $N_{H}^{*}(s, N)=N$ and $N_{H}^{*}(s, N)=N$. First consider $a=a_{H}$. Because $V(k) \geqslant \widetilde{u}\left(s_{L}, c_{H}+\gamma(k)\right)$, a sufficient condition for $N_{H}^{*}(s, N)=N$ is:

$\widetilde{u}\left(s_{L}, c_{H}+\gamma(k)\right) \leqslant(1-p) \cdot \widetilde{u}\left(s_{H}, c_{H}+\gamma(k+1)\right)+p \cdot \widetilde{u}\left(s_{L}, c_{H}+\gamma(k+1)\right)$,

for all $k=1, \ldots, N-1$, or, equivalently,

$$
e^{-\rho\left(s_{L}-c_{H}-\gamma(k)\right)} \geqslant(1-p) \cdot e^{-\rho\left(s_{H}-c_{H}-\gamma(k+1)\right)}+p \cdot e^{-\rho\left(s_{L}-c_{H}-\gamma(k+1)\right)} .
$$

This is equivalent to

$$
\max _{k \in\{1, \ldots, N-1\}}\left\{e^{\rho(\gamma(k+1)-\gamma(k))}\right\} \leqslant \frac{1}{(1-p) \cdot e^{-\rho\left(s_{H}-s_{L}\right)}+p} .
$$

Likewise, a sufficient condition for $N_{L}^{*}(s, N)=N$ is

$$
\max _{k \in\{1, \ldots, N-1\}}\left\{e^{\rho(\gamma(k+1)-\gamma(k))}\right\} \leqslant \frac{1}{(1-q) \cdot e^{-\rho\left(s_{H}-s_{L}\right)}+q} .
$$

Now take any $(i, j)$ with $i<N$ and/or $j<N$. We will show that (27) implies that there does not exist a contract that induces the manager to deliver high effort, and that satisfies $N_{H}^{*}(s, N)=i$ and $N_{L}^{*}(s, N)=j$. Suppose such a contract does exist. Let $B(i, j)$ denote the minimum level of the bonus needed to motivate the manager to deliver high effort, given that he chooses $\bar{n}=i$ if $a=a_{H}$, and $\bar{n}=j$ if $a=a_{L}$. Then, a contract that induces the manager to deliver high effort and to choose $N_{H}^{*}(s, N)=i$ and $N_{L}^{*}(s, N)=j$ needs to satisfy $s_{H}-s_{L} \geqslant B(i, j)$.

Now suppose (27) is satisfied. Then,

$$
\begin{aligned}
\max _{k \in\{1, \ldots, N-1\}}\left\{e^{\rho(\gamma(k+1)-\gamma(k))}\right\} & \leqslant \frac{1}{(1-q) \cdot e^{-\rho \cdot B(i, j)}+q} \\
& \leqslant \frac{1}{(1-q) \cdot e^{-\rho\left(s_{H}-s_{L}\right)}+q} \\
& \leqslant \frac{1}{(1-p) \cdot e^{-\rho\left(s_{H}-s_{L}\right)}+p} .
\end{aligned}
$$

Therefore, (45) and (46) are satisfied, and it follows that $N_{H}^{*}(s, N)=N_{L}^{*}(s, N)=$ $N$, i.e., the manager has an incentive to determine all $N$ signals. This contradicts $N_{H}^{*}(s, N)=i$ and $N_{L}^{*}(s, N)=j$. Thus, for any $(i, j)$ with $i<N$ and/or $j<N$, the agent cannot be motivated to deliver high effort and choose $\bar{n}=i$ if $a=a_{H}$, and $\bar{n}=j$ if $a=a_{L}$, and so the optimal contract must satisfy $N_{H}^{*}(s, N)=N_{L}^{*}(s, N)=N$.

\section{References}

Arya A, Glover J, Sunder S (1998) Earnings management and the revelation principle. Rev Account Stud $3: 7-34$ 
Arya A, Young RA, Woodlock P (1992) Managerial reporting discretion and the truthfulness of disclosures. Econ Lett 39:163-168

Demski JS (1998) Performance measure manipulation. Contemp Account Res 15(3):261-285

Demski JS, Frimor H, Sappington DEM (2004) Efficient manipulation in a repeated setting. J Account Res 42(1):31-49

Demski JS, Patell JM, Wolfson MA (1984) Decentralized choice of monitoring systems. Account Rev 59(1):16-34

Dye RA, Magee RP (1991) Discretion in reporting managerial performance. Econ Lett 35:359-363

Grossman SJ, Hart OD (1983) An analysis of the principal-agent problem. Econometrica 51(1):7-45

Holmström B (1979) Moral hazard and observability. Bell J Econ 10(1):74-91

Lewis TR (1980) Bonuses and penalties in incentive contracting. Bell J Econ 11:292-301

Liang PJ (2004) Equilibrium earnings management, incentive contracts, and accounting standards. Contemp Account Res 21(3):685-717

Mookherjee D, Png I (1989) Optimal auditing, insurance, and redistribution. Q J Econ 104(2):399-415

Ozbilgin M, Penno M (2008) The assignment of decision rights in formal information systems. J Manag Account Res 20:83-105

Penno M (2005) The contracting value of tainted reports in cost reduction settings. Eur Econ Rev 49:1979_ 1985

Rasmusen E (1987) Moral hazard in risk-averse teams. RAND J Econ 18(3):428-435

Verrecchia RE (1986) Managerial discretion in the choice among financial reporting alternatives. J Account Econ 8:175-195 